\title{
Summertime Planetary Wave Resonance in the Northern and Southern Hemispheres $\mathscr{A}$
}

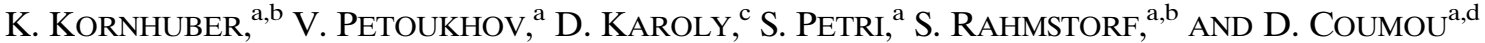 \\ ${ }^{\text {a }}$ Potsdam Institute for Climate Impact Research-Earth System Analysis, Potsdam, Germany \\ ${ }^{\mathrm{b}}$ Universität Potsdam, Potsdam, Germany \\ ${ }^{\mathrm{c}}$ ARC Centre of Excellence for Climate System Science, University of Melbourne, Parkville, Victoria, Australia \\ ${ }^{\mathrm{d}}$ Institute for Environmental Studies, Vrije Universiteit, Amsterdam, Netherlands
}

(Manuscript received 29 September 2016, in final form 13 April 2017)

\begin{abstract}
Slow-moving planetary waves of high amplitudes are often associated with persistent surface weather conditions. This persistence can lead to extreme weather events with potentially serious implications for society and nature. Quasi-resonant amplification (QRA) of planetary waves has been proposed as a mechanism to generate high-amplitude hemisphere-wide patterns of wavenumbers 6-8 in the Northern Hemisphere (NH) summer. Here this mechanism is studied in both hemispheres. Analyzing 1979-2015 reanalysis data, evidence for QRA in the Southern Hemisphere ( $\mathrm{SH}$ ) is found for wavenumbers 4 and 5. It is shown that the difference in resonating wavenumbers between hemispheres stems from the different magnitude and latitudinal shape of the respective zonal-mean zonal winds. During resonance events a strong and narrow jet is observed in both hemispheres, whereas the emergence of a second zonal mean jet at high latitudes (i.e., a "double jet") is seen in the NH only. Strong and narrow jets can form stable, zonally oriented waveguides, which are an essential prerequisite for resonance. After investigating the waves' preferred phase positions during QRA, a bimodal behavior is identified for wavenumbers 4 and 5 in the SH and for wavenumber 6 in the $\mathrm{NH}$, whereas wavenumbers 7 and 8 exhibit a single preferred phase position in the NH. Composite plots of meridional wind and temperature anomalies during periods when the resonating wave is in the preferred phase position indicate those regions that are most likely to experience heat extremes. These regions include western North America, western Europe, and central Eurasia in the NH and Patagonia and Australia in the SH.
\end{abstract}

\section{Introduction}

Quasi-stationary Rossby waves have a pronounced influence on weather in the midlatitudes in both the Southern and Northern Hemisphere ( $\mathrm{SH}$ and $\mathrm{NH}$, respectively) and are often associated with extreme weather events (Hoskins and Woollings 2015; Screen and Simmonds 2014; Coumou et al. 2014; Woollings 2010). Rossby or planetary waves are large-scale oscillations of the midlatitude flow resulting from the latitudinal dependency of the Coriolis effect combined with thermal or orographic perturbations (Hoskins and Karoly 1981; Branstator 1983; Hoskins and Ambrizzi 1993; Frederiksen and Webster 1988). Rossby (1939) suggested that the

\footnotetext{
Supplemental information related to this paper is available at the Journals Online website: http://dx.doi.org/10.1175/JCLI-D-160703.s1.
}

Corresponding author: K. Kornhuber, kai.kornhuber@ pik-potsdam.de midlatitudinal tropospheric circulation varies between two regimes characterized by a zonal type with dominant westerly currents and a meridional type with weak zonal flow and pronounced north-south meanders. In the meridional-type flow pattern, weather patterns are more likely to persist longer over specific regions. If these patterns last for several days or weeks this might lead to extreme events such as heat waves and droughts or cold spells and floods (Black et al. 2004; Lau and Kim 2012; Ogi et al. 2005). In such situations, cold air can be carried far south while warm air can reach northern regions, thus leading to unusual local temperature conditions (Petoukhov et al. 2013; Screen and Simmonds 2014; Coumou et al. 2014; Hoskins and Woollings 2015; Teng et al. 2013; Branstator 2002). One way to quantify planetary waves mathematically is to decompose the midlatitude meridional wind field into its spectral components. Waves are characterized by their amplitude (north-south wind speed) and phase speed (velocity of west-east propagation) (Rossby 1940; Charney and Eliassen 1949; Haurwitz 1940a; Haurwitz 1940b) 
and can interact with each other as well as with the background flow (Frederiksen and Webster 1988). They can be subject to reflection, diffraction, dispersion, and resonance (Hoskins and Karoly 1981).

One way to generate quasi-stationary Rossby waves is via the amplification of waves trapped in waveguides, generating circumglobal wave patterns (Branstator 2002). A waveguide leads to situations with little wave dissipation, due to high reflectivity at its latitudinal boundaries. This prevents the loss of wave energy through meridional dispersion. Waveguides thereby constitute favorable pathways for wave propagation and/or the generation of persistent stationary modes that may become apparent as atmospheric teleconnections (Branstator 2002; Branstator 1983; O'Kane et al. 2016; Hoskins and Karoly 1981; Ambrizzi et al. 1995; Held 1983; Dionne et al. 1988; Wu 1993; Teng et al. 2013). In their pioneering work, Charney and De Vore (1979) found three equilibria for orographically forced Rossby waves in a simple barotropic channel model, of which two were stable: a high-index (resonant) blocking-type state with strong waves, and a low index (nonresonant) zonal state. Because of limitations of the applied channel model, as well as their prescribed external forcing, it was unclear to what extent their findings were quantitatively applicable to the real atmosphere.

Several studies analyzed the response of the planetary wave pattern to different jet shapes and different positions of orographic and/or thermal forcing but most studied winter circulation only. Based on $\mathrm{NH}$ winter circulation, Branstator (2002) found a circumglobal wavenumber-5 pattern confined to the positions of the storm tracks. A similar pattern was identified by Lutsko and Held (2016) in an analysis of the role of orography in an idealized dry climate model. Analyzing SH summer and $\mathrm{NH}$ winter circulation, Manola et al. (2013), using a channel model similar to Charney and De Vore (1979), found circumglobal wavenumber-3-5 patterns and showed that stronger and narrower jets lead to amplified zonal waves, with wavelength increasing with jet strength. The existence of forced resonant states in a spherical model was shown by Yang et al. (1997), who prescribed zonal waveguides by a change of direction in the zonal winds from westerlies to easterlies at the subtropical and subpolar latitudinal boundaries, thereby confining a midlatitudinal wave. Zidikheri et al. (2007) generalized Charney and De Vore's (1979) theory of atmospheric multiequilibria in several important aspects, such as the effects of transient eddies and more realistic topography on the system dynamics, and the possibility of resonant multiequilibria on a spherical domain. They found two separated regimes in both $\mathrm{NH}$ and $\mathrm{SH}$ for zonal wavenumber 2. However, the zonal winds necessary for creating those equilibria were unrealistically high. The majority of the aforementioned studies analyzed quasi-stationary resonant waves in winter with zonal wavenumbers lower than 6 .

For NH summers, Petoukhov et al. (2013) showed how a synoptic-scale free wave trapped in a midlatitude waveguide can resonate with the slow-moving forced wave and thereby increase its amplitude through a quasi-resonant amplification (QRA). The concept of the stationary wavenumber $K_{s}$ assumes that the dimensional zonal wavenumber $K$ is constant on a ray along which the wave's energy propagates, while the meridional wavenumber $l$ varies such that $K_{s}^{2}=K^{2}+l^{2}$ holds (Hoskins and Karoly 1981; Hoskins and Woollings 2015). Assuming circumglobal waves, waveguide conditions based on a realistic zonally symmetric background flow for synoptic-scale wavenumbers were determined. Starting from the quasilinear barotropic vorticity equation on a sphere (Pedlosky 1979; Hoskins and Karoly 1981), Petoukhov et al. (2013) formulated a theoretical framework for the trapping and amplification of quasi-stationary synoptic scale waves (zonal wavenumber 6 and higher), which were earlier considered to be overwhelmingly transient (Dell'Aquila et al. 2005; Lucarini et al. 2007). High-amplitude quasistationary waves with these wavenumbers manifest themselves as a longitudinally oriented hemisphere-wide chain of persistent meridional-type patterns in the $\mathrm{NH}$ midlatitudes (for further details see section 2). Such circulation patterns were observed during several recent extreme summer weather events such as the European heat wave of 2003, the Moscow heat wave of 2010, the Balkan floods of 2013, and the European heat wave of 2015 (Petoukhov et al. 2013; Coumou et al. 2014; Kornhuber et al. 2017; Stadtherr et al. 2016; Petoukhov et al. 2016).

In a detailed statistical analysis of the 1979-2015 NH summers, Kornhuber et al. (2017) showed that when resonance conditions are fulfilled, the midlatitude circulation is characterized by slow-moving, high-amplitude waves with wavenumbers 6-8. Moreover, QRA conditions were met during a third of all incidents of high-amplitude wavenumbers $6-8$ [defined by amplitudes exceeding 1.5 standard deviations $(1.5 \sigma)$ above the climatological mean]. Thus in the NH, QRA is an important mechanism for creating quasi-stationary high-amplitude waves in summer, although certainly not the only mechanism.

Here we expand these analyses to address the following research questions:

- Does QRA occur in the SH as well, and if so, what are the relevant wavenumbers?

- Does the magnitude or shape of the zonal-mean zonal wind profile determine waveguide formation?

- At what latitudes are waveguides most likely to form?

- Do resonating waves have preferred phase positions? 
The last question can give insight into which regions are most likely to experience extreme hot or cold conditions during QRA events.

We outline the theoretical background for the QRA mechanism in the methods section (section 2) before providing details on data and analytical tools. In the analysis section (section 3 ) we estimate the range of free waves to be considered for QRA based on the circulation climatology in each hemisphere. We then analyze the influence of the strength and shape (in terms of derivatives) of the mean zonal wind on waveguide formation. We examine the difference in wave speed and amplitude of the considered waves during QRA events compared to non-QRA days. By analyzing the preferred phase positions of the considered wavenumbers, we determine the locations of negative and positive temperature anomalies during QRA events. Summarizing remarks are provided in the discussion and conclusions section (section 6).

\section{Methods}

\section{a. Detection of quasi-resonant amplification of planetary waves}

QRA of a forced planetary wave with nondimensional wavenumber $m$ requires a synoptic-scale free wave with zonal nondimensional wavenumber $k \approx m$ to be trapped in a midlatitude waveguide. When a waveguide exists and the combined orographic and thermal forcing pattern is sufficiently large, then QRA can create a high-amplitude quasi-stationary planetary wave with wavenumber $m$ (Petoukhov et al. 2013). Thus, the occurrence of QRA of wavenumber $m$ boils down to two criteria:

(i) A waveguide for a free wave with wavenumber $k \approx m$ is present.

The formation of a waveguide in the zonal mean depends solely on the square of the dimensional meridional wavenumber $l$, which itself is a function of the zonal-mean zonal wind $U$, latitude $\varphi$, and $k$ only:

$$
\begin{aligned}
l^{2}= & \frac{2 \Omega \cos ^{3} \varphi}{a U}-\frac{\cos ^{2} \varphi}{a^{2} U} \frac{d^{2} U}{d \varphi^{2}}+\frac{\sin \varphi \cos \varphi}{a^{2} U} \frac{d U}{d \varphi} \\
& +\frac{1}{a^{2}}-\left(\frac{k}{a}\right)^{2},
\end{aligned}
$$

where $a$ is Earth's radius and $\Omega$ its angular velocity. In Petoukhov et al. (2013), Eq. (1) is derived from the quasi-linear barotropic vorticity equation on a sphere (Pedlosky 1979; Hoskins and Karoly 1981) for adiabiatic free waves at the equivalent barotropic level (EBL, i.e., about $300-500 \mathrm{hPa}$ and zero right-hand side, thus no forcing). In the Mercator projection of the sphere this equation can be written as

$$
\left(\frac{\partial}{\partial t}+\frac{U}{\cos \varphi} \frac{\partial}{\partial x}\right)\left(\frac{\partial^{2} \psi}{\partial x^{2}}+\frac{\partial^{2} \psi}{\partial y^{2}}\right)+\beta \frac{\partial \psi}{\partial x}=0,
$$

where $\psi$ is the azonal streamfunction at the EBL; $x=$ $a \lambda$, where $\lambda$ is the longitude; $y=a \ln [(1+\sin \varphi) / \cos \varphi]$; $t$ is time; and

$$
\beta=\frac{2 \Omega}{a} \cos ^{2} \varphi-\frac{d}{d y} \frac{1}{\cos ^{2} \varphi} \frac{d}{d y}\left(\frac{U}{\cos \varphi} \cos ^{2} \varphi\right) .
$$

For plane wave solutions, $\psi=\exp [i(k / a) x+l y-\omega t]$, the dispersion relation to Eq. (2) is

$$
\begin{aligned}
\omega= & \frac{U}{\cos \varphi} K-\frac{K}{K^{2}+l^{2}}\left[\frac{2 \Omega}{a} \cos ^{2} \varphi\right. \\
& \left.-\frac{d}{d y} \frac{1}{\cos ^{2} \varphi} \frac{d}{d y}(\cos \varphi)\right] .
\end{aligned}
$$

As Eq. (3) does not explicitly depend on $x$ and $t$, the dimensional zonal wavenumber $K=k / a$ and the frequency $\omega$ are constant on a ray along which the wave energy propagates, while the meridional wavenumber $l$ varies continuously with latitude so that $K_{s}^{2}=K^{2}+l^{2}$ holds (Whitham 1960; Hoskins and Karoly 1981). We now only consider quasistationary waves $(\omega \approx 0)$ and assume the validity of the Wentzel-Kramers-Brillouin method (Dingle 1973; Murdock 1987). For these waves the group velocity is expressed as

$$
c_{g}=2 \frac{K}{K_{s}} \frac{U}{\cos \varphi},
$$

with the dimensional "stationary wavenumber" $K_{s}=\beta \cos \varphi / U$. Employing the aforementioned alternative expression $K_{s}^{2}=K^{2}+l^{2}$ (Hoskins and Karoly 1981) we obtain $l^{2}=\beta \cos \varphi / U-K^{2}$, which can be converted to Eq. (1).

A midlatitude waveguide is defined as such when two turning points (TPs) appear within the midlatitudes with $l^{2}>0$ and $U>0$ in between the TPs and also $U>0$ in the vicinity of the TPs. In such a case the waves are strongly reflected at the midlatitudinal TPs with a near-zero leakage of the waves' energy toward the equator or poles. In that respect $l^{2}$ can be interpreted as a refractive index: in fact, it can alternatively be derived from the barotropic refractive index introduced by Branstator (1983) assuming circumglobal waves of high wavenumbers in the midlatitudes at realistic values of 
tropospheric and near-surface "eddy friction" (see the discussion of barotropic refraction index in the supplemental material).

(ii) The combined amplitude of thermal and orographic forcing (effective forcing) for a forced wave with wavenumber $m$ is within the dominating wavenumbers of the ruling forcing spectrum.

Here forced waves differ from free waves by the inclusion of an effective wave forcing term $F_{\text {eff }}$ on the right-hand side of the wave equation [see Eq. (S1c) in Petoukhov et al. (2013)]. This forcing term is a combination of thermal and orographic forcing:

$$
F_{\text {eff }}=\frac{2 \Omega \sin \varphi \cos (\varphi)^{2}}{a T_{c}} \frac{\partial \hat{T}}{\partial \lambda}-\frac{2 \Omega \sin \varphi \cos (\varphi)^{2}}{a H} \kappa \frac{\partial h_{\text {or }}}{\partial \lambda},
$$

where $T_{c}=200 \mathrm{~K}$ is a constant reference temperature at the equivalent barotropic level (EBL), $\hat{T}$ is the azonal temperature at $300 \mathrm{hPa}, H=12000 \mathrm{~m}$ is the characteristic scale of the troposphere height, $\kappa$ is the characteristic value of the ratio of the zonally averaged zonal wind $U$ at $300 \mathrm{hPa}$ and the zonally averaged zonal wind at the mean orographic height $U_{\text {or }}\left(\kappa=U_{\text {or }} / U\right)$, and $h_{\text {or }}$ is the height of the coarse-resolution orography (see Fig. S18 in the supplemental material; Held et al. 1985). In Eq. (5) thermal forcing is expressed by the first term, whereas the second term in Eq. (5) represents the orographic forcing. The azonal temperature field contains the temperature deviations from the given zonal mean profile. It is determined by subtracting the zonal mean temperature field from the actual temperature field at each time step.

For amplification of the trapped wave a minimum amount of forcing is required, as can be seen from the amplitude equation below [Eq. (6) herein, or Eq. (3) in Petoukhov et al. (2013)]. When a waveguide is present for a free synoptic wave with wavenumber $k$ [i.e., criterion (i) is fulfilled] then

$$
A_{m}=\frac{A_{\mathrm{eff}}}{\sqrt{\left[(k / a)^{2}-(m / a)^{2}\right]^{2}+\left(L / a^{2}+R^{2} / L\right)^{2}(m / a)^{2}}}
$$

is valid. Here, $A_{m}$ is the amplitude of the trapped wave; $m$ is the wavenumber of the forcing wave; $R=\kappa R_{0}$ and $L=\kappa L_{0}$, where $R_{0}=0.135$ is the Rossby number, and $L_{0}=6 \times 10^{5} \mathrm{~m}$ the Rossby radius of the eddies dominantly contributing to atmospheric and near-surface friction (see the supplemental material for estimation of the Rossby number $R_{0}$ and the Rossby radius $L_{0}$ ); and $A_{\text {eff }}$ is the amplitude of $F_{\text {eff }}$ [Eq. (5)]. The $A_{\text {eff }}$ is calculated by applying a zonal FFT on the area-weighted meridional average of $F_{\text {eff: }}$ :

$$
A_{\text {eff }}=\operatorname{FFT}\left(F_{\text {eff }}\right) .
$$

Equation (6) shows that the zonal wavenumber $k$ needs to be close to the zonal wavenumber $m$ of the forced wave. In that case the first term in the denominator is close to zero, resulting in a strong amplification. Thus, resonance results in much larger wave amplitudes than can be expected from the effective forcing $A_{\text {eff }}$ alone (see also the supplemental material on effective forcing amplitude). This is the essence of resonant amplification. With criteria (i) and (ii) fulfilled, QRA can theoretically occur given the assumptions and simplifications entering the equation [see Kornhuber et al. (2017) for a detailed discussion]. Apart from $k$ being close to $m$, for efficient amplification to take place, $A_{\text {eff }}$ has to be of certain magnitude. Therefore, $A_{\text {eff }}$ is determined for wavenumbers $m=1-15$ and a threshold quantile is estimated for each time step respectively. In our analysis we set this threshold to the median forcing, implying that the forcing for the trapped wave has to be among the strongest $50 \%$ for that particular time step. As a test, we check whether the predicted wave amplitude [from Eq. (6)] is close to that observed during periods when both resonance conditions are fulfilled [(i) + (ii)]. We calculate $A_{m}$ for $k=m \pm 0.2$, which results in an estimated range of wave amplitudes expected from resonance. When the observed wave amplitude falls within this range, the amplitude test (AT) is passed (see Kornhuber et al. 2017; see also the discussion of amplitude test in the supplemental material).

\section{b. Parameter estimation}

Although the waveguide criterion (i). [see Eq. (1)] does not contain any hemisphere-dependent parameters, some enter in Eqs. (5)-(7). Those equations are linked to QRA criterion (ii) and the AT only and not to waveguide formation. One of those parameters is $\kappa$, the ratio between the zonal wind $U$ at $300 \mathrm{hPa}$ and the zonalaveraged zonal wind at the average zonal orographic height $U_{\text {or }}\left(\kappa=U_{\text {or }} / U\right)$. Using climatological zonalmean zonal winds, this measure was estimated to be $\kappa_{\mathrm{NH}} \approx 0.4$ in the NH (Petoukhov et al. 2013; Charney and Eliassen 1949; Held 1983; Held et al. 1985). In the SH midlatitudes this ratio is larger because of less orographic friction, and it was estimated to be $\kappa_{\mathrm{SH}} \approx 0.7$. Entering the equation for the effective forcing [Eq. (5)] and the expected amplitude [Eq. (6)], this parameter 
affects resonance criterion (ii). and therefore the AT. We tested the sensitivity of the exact value of $\kappa$ on our resonance detection scheme and its impact was shown to be marginal (see Figs. S4 and S5 and Table S1 in the supplemental material).

Other parameters in Eqs. (5)-(7) were set to the same value for both $\mathrm{NH}$ and $\mathrm{SH}$ analyses. Those are $T_{c}=$ $200 \mathrm{~K}$, a constant reference temperature at the EBL, and $H=12000 \mathrm{~m}$, the characteristic scale of the troposphere height (Held et al. 1985).

We defined the extension of the midlatitudinal belt based on the range of positive $U$ values in the zonal average. In the $\mathrm{NH}$ this range was set as $37.5^{\circ}-57.5^{\circ} \mathrm{N}$. In the $\mathrm{SH}$ the climatological profile of $U$ exhibits values above $10 \mathrm{~m} \mathrm{~s}^{-1}$ closer to the equator (Pena-Ortiz et al. 2013; Trenberth 1991). Therefore, the latitudinal range was set to $30^{\circ}-60^{\circ} \mathrm{S}$. The definition of the midlatitudinal range is employed for the meridional averaging of the $v$-wind field (wave speed and amplitude) and for the temperature and orography, when calculating the effective forcing amplitude [Eq. (5)]. It thereby impacts criterion (ii) and the AT (Table S2 in the supplemental material). Wave amplitudes and phase speeds are higher when averaging over higher latitudes (Fig. S6) (lower latitudes show a higher fraction of landmass, compared to higher latitudes in the $\mathrm{SH})$. The QRA detection, however, turned out to be robust against different choices of latitudinal ranges (Figs. S6 and S7 in the supplemental material), with only minor differences in results. Criteria (i) and (ii) are tested on zonal wavenumbers $k=m \pm 0.2$ with a step size of $\Delta k=0.1$ resulting in values of $k$ per integer wavenumber $m$. Setting a threshold on the number of $k$ per $m$ that have to fulfill the respective condition serves as a noise filter. The task consists of finding a value (between 1 and 5) that rejects noise but still captures important events. We use a Kolmogorov-Smirnov test applied on the probability density distributions of quasi-stationary waves during QRA days and non-QRA days (see Figs. S2 and S3 in the supplemental material) to indicate the best choice. In the $\mathrm{NH}$ a value of 4 was found to deliver the best results. In the $\mathrm{SH}$ a value of 2 was found to be sufficient.

\section{c. Data}

The ERA-Interim reanalysis datasets of summer months of years 1979-2015 were analyzed (Dee et al. 2011). Summer months were set as June-August (JJA) in the $\mathrm{NH}$ and December-February (DJF) in the SH. Daily data of temperature and zonal and meridional wind fields were analyzed at a pressure level of $300 \mathrm{hPa}$ on a $2.5^{\circ} \times 2.5^{\circ}$ grid. Daily temperature anomalies are determined by a gridpointwise subtraction of respective daily linear trends.
A 15-day moving average was applied on temperature and wind fields. Applying a running average serves the purpose of a low-pass filter (Fig. S20 in the supplemental material). This is necessary to filter out fast-moving transients. A 15-day running mean was chosen because it states the approximate time scale of the phenomena we are interested in (Petoukhov et al. 2013; Kornhuber et al. 2017). Thus, applying a 15-day running mean serves as a low-pass filter, not a bandpass filter as, for example, in Schneider et al. (2015) (see Fig. S20). The results are independent of the exact choice of runningmean averaging (see the supplemental material for sensitivity analysis-running-mean averaging).

Following prior analysis by Petoukhov et al. (2013) and Kornhuber et al. (2017), orographic data were taken from Hastings and Dunbar (1999). Taking into account that the resulting forcing in the troposphere is much smoother than orography itself, the latter was coarsened to a $10^{\circ} \times 15^{\circ}$ grid as in Charney and Eliassen (1949) (Fig. S10 in the supplemental material). Planetary wave amplitudes were determined from reanalysis data by applying a zonal fast Fourier transform (FFT) on the area-weighted meridional mean from $37.5^{\circ}$ to $57.5^{\circ} \mathrm{N}$ (from $30^{\circ}$ to $60^{\circ} \mathrm{S}$ ) of daily meridional wind data and 15-day running-mean meridional wind data respectively. We calculated the phase speed (eastward propagation) by applying a fourth-order accurate numerical approximation of the transient derivative of the waves' phase (Coumou et al. 2014).

\section{Analysis}

\section{a. Relevant zonal wavenumbers}

QRA is an amplifying mechanism effective for free waves with a normally weak quasi-stationary component. In NH summer (JJA) those waves typically exhibit zonal wavenumbers $k \geq 6$ (Petoukhov et al. 2013; Coumou et al. 2014). When studying the validity of the QRA mechanism under dynamic conditions that differ from the NH JJA circulation, the first task is to determine the range of relevant waves with weak quasi-stationary components. The linear Rossby wave equation relates phase speed $c$ to zonal winds $U$ (Rossby 1939):

$$
c=U-\beta /\left(K^{2}+l^{2}\right),
$$

where $\beta$ is the Rossby parameter, and $K$ and $l$ are the zonal and meridional wavenumber, respectively. This equation shows that the phase speed of free traveling waves is linearly dependent on the magnitude of the zonally averaged zonal winds: stronger zonal winds lead to lower wavenumbers exhibiting quasi-stationarity. To 

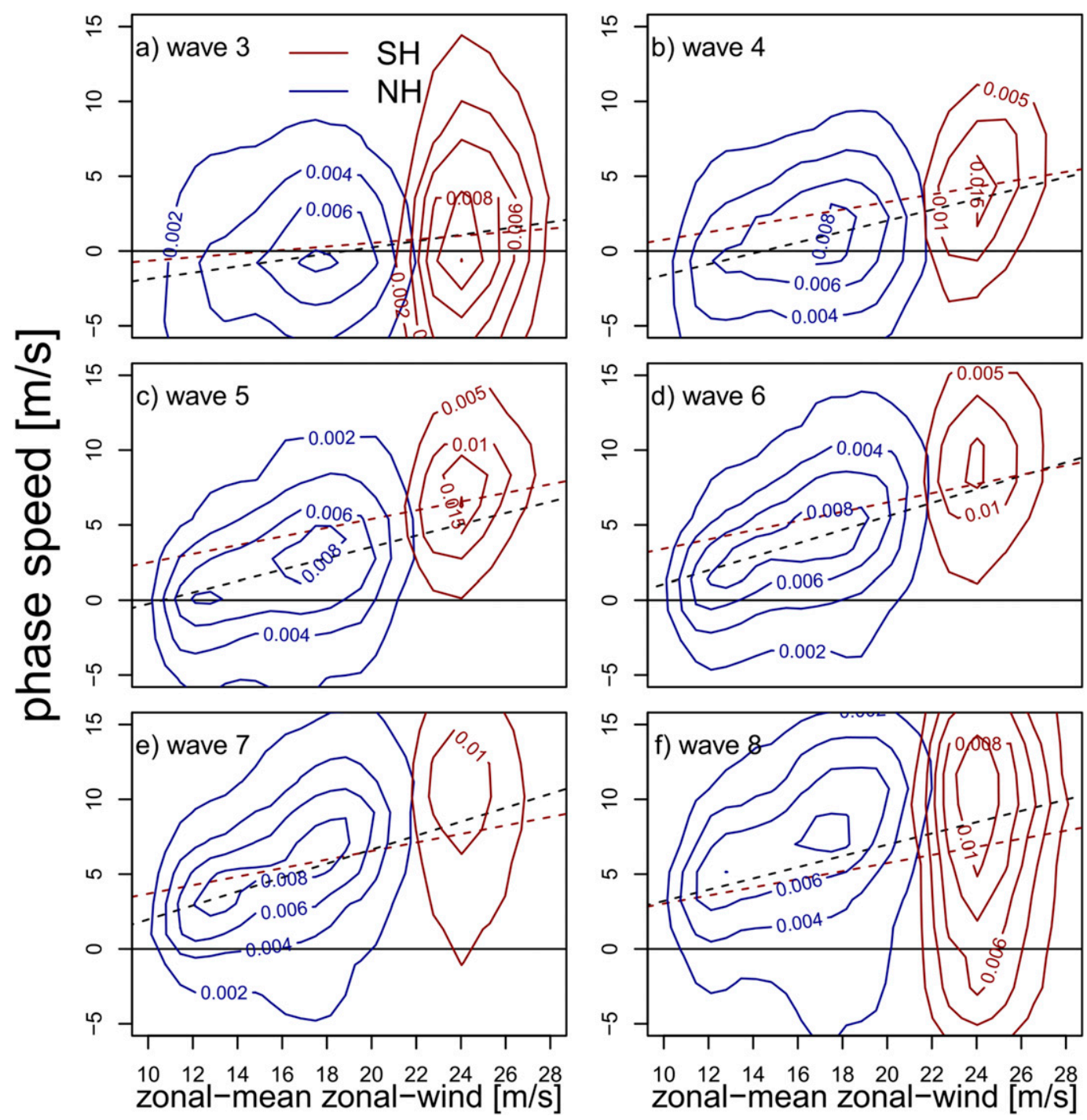

FIG. 1. Annual probability density distributions for daily values of the zonal-averaged $U$ and the wave phase speed $c$ at $300 \mathrm{hPa}$ in the midlatitudes of the $\mathrm{SH}\left(30^{\circ}-60^{\circ} \mathrm{S}\right.$; red) and the $\mathrm{NH}\left(37.5^{\circ}-57^{\circ} \mathrm{N}\right.$; blue $)$ of wavenumbers $3-8$ for all calendar years (1979-2015). In the SH wavenumbers $\geq 4$ show predominantly eastward propagation (positive $c$ ), whereas in the $\mathrm{NH}$ this holds for wavenumbers $\geq 6$.

investigate the wave properties in both hemispheres we plot the 2D probability density distribution of zonally averaged zonal wind speeds $U$ and the phase speeds of wavenumbers 3-8 in the midlatitudes (Fig. 1). Values of $U$ were determined by longitudinally averaging the mean daily values of the zonal wind speeds in the midlatitudinal belt $\left(30^{\circ}-60^{\circ} \mathrm{S}\right.$ and $\left.37.5^{\circ}-57.5^{\circ} \mathrm{N}\right)$ at the 300-hPa pressure level. The distributions given in Fig. 1 show the approximate linear relationship with $U$ expressed by a linear fit (NH: blue dashed line, $\mathrm{SH}$ : red dashed line). The $\mathrm{NH}$ shows a larger variability in $U$ due to stronger seasonal differences (see also Fig. S1 in the supplemental material). In the $\mathrm{SH}$ wind speeds are generally higher, values ranging between 20 and
$26 \mathrm{~m} \mathrm{~s}^{-1}$, while $U$ exhibits a lower seasonal variability (Peixoto and Oort 1992). As stated above, wavenumbers below 6 are mostly stationary in the NH. This holds especially for the summer season with its weaker zonal mean wind (Fig. S1). In the SH, the probability density distribution of wavenumber 3 shows a largely quasistationary behavior while also containing eastward- and westward-propagating waves (positive and negative values of $c$, respectively). Wavenumbers above 3 are found to predominantly propagate eastward with a small stationary component. These results suggest that the range of free waves to which the QRA mechanism is potentially applicable in the $\mathrm{SH}$ consists of wavenumbers 4 and higher. Thus, in contrast to the $\mathrm{NH}$, 

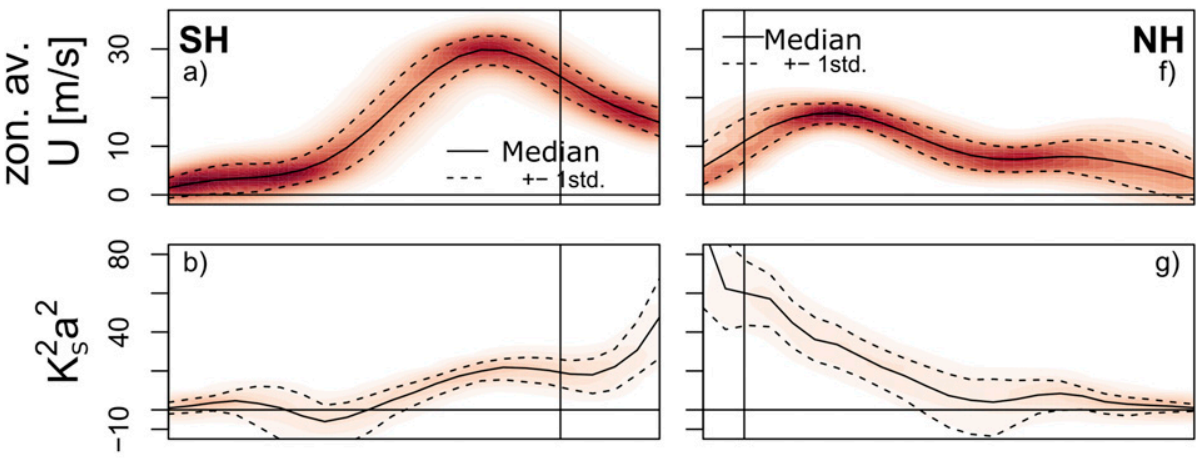

g)
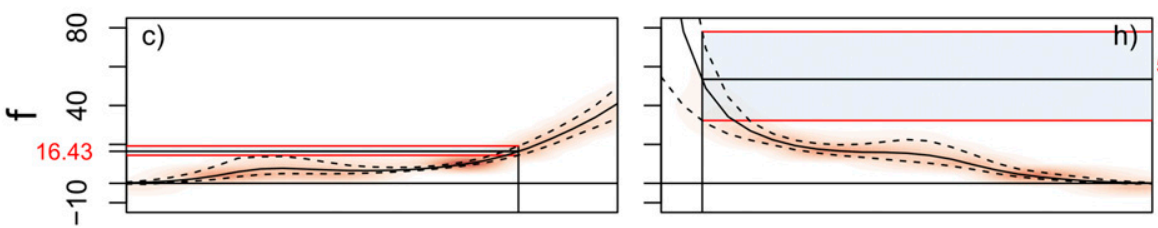

)

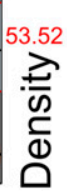

రั
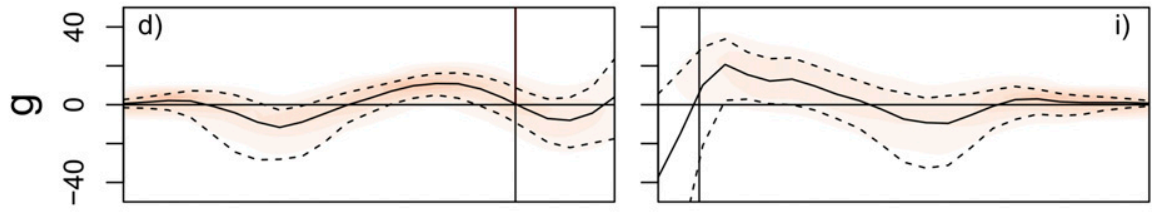

i)

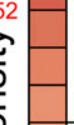

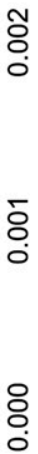
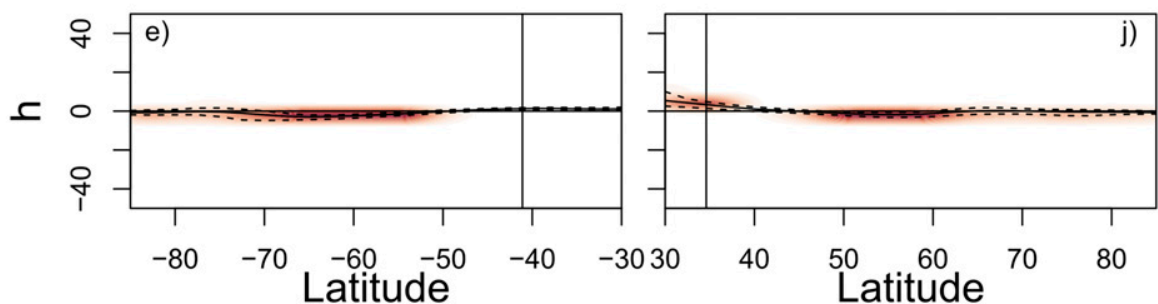

FIG. 2. (a) The 15-day running mean of $U$ as DJF climatology (black) and variability is shown as a probability density (red shading). The median is shown as a solid black line and the $\pm 1 \sigma$ marks are depicted with black dashed lines respectively. (b),(g) Density of $K_{s}^{2} a^{2}$, (c),(h) first term $f(\varphi)$ determining the general height, (d),(i) second $g(\varphi)$ term determining the waveguide positons during QRA days, and (e),(j) third term $h(\varphi)$. The mean position of the equatorward TP derived from (g) is indicated by a vertical black line. In (c) and (h), the mean value of $f(\varphi)$ at these latitudes is given in red at the left and right side of the respective panels, while the horizontal red lines denote its uncertainty range based on its $\pm 1 \sigma$ range.

where the QRA mechanism has been derived for wavenumbers 6 and higher, lower wavenumbers should be considered to test for QRA in the SH summer due to the comparably stronger zonal winds.

Based on these results we explore the behavior of the squared meridional wavenumber $l^{2}$ [Eq. (1)] in each hemisphere. We put an emphasis on hemispheric differences in waveguide formation.

\section{b. Climatology of the squared meridional wavenumber}

Waveguide formation in the midlatitudes is the first and foremost precondition for QRA to occur. The occurrence of a waveguide depends on the shape of the meridional wavenumber $l^{2}$, which is a function of latitude $\varphi$ and the zonally averaged zonal wind $U$ [Eq. (1)]. As $U$ enters the denominators of the righthand side of Eq. (1), a stronger zonal flow leads generally to smaller values of $l^{2}$, but because of the existence of first- and second-order derivatives the shape of $U$ also matters (Figs. 2a,f). In the NH, one pronounced peak in $U$ is observed at $40^{\circ} \mathrm{N}$ and a secondary peak is positioned at $70^{\circ} \mathrm{N}$. The SH climatological zonal flow is characterized by a broader and stronger single jet with the strongest wind speeds at $50^{\circ} \mathrm{S}$, about twice as strong as those in the NH.

In the following we will investigate how these climatological differences in $U$ affect the formation of waveguides by taking a closer look at the shape and subterms of $l^{2}(\varphi, U)$. Starting from Eq. (1) we multiply both sides 
by $a^{2}$, add $k^{2}$, and obtain an expression for the stationary wavenumber $K_{s}^{2} a^{2}$ :

$$
K_{s}^{2} a^{2}=(l a)^{2}+k^{2}=f(\varphi)+g(\varphi)+h(\varphi)+1,
$$

where

$$
\begin{aligned}
& f(\varphi)=\frac{2 \Omega \cos ^{3} \varphi a}{U}, \\
& g(\varphi)=-\frac{\cos ^{2} \varphi}{U} \frac{d^{2} U}{d \varphi^{2}}, \text { and } \\
& h(\varphi)=\frac{\sin \varphi \cos \varphi}{U} \frac{d U}{d \varphi} .
\end{aligned}
$$

As Eq. (3) is independent of $x$ and $t, k$ is constant on a ray along which the wave energy travels, while $l$ changes so that Eq. (9) holds (see section 2a). Thus $k$ is only an additive constant without any influence on the shape of $l^{2}$ Thus, while it is our aim to explore the general features of $l^{2}$ it is sufficient and convenient to investigate $K_{s}^{2} a^{2}$. We can then plot $K_{s}^{2} a^{2}$ and determine the shift that is applied by a certain value of $k$ along the $y$ axis. To assess the behavior of each component we plot $K_{s}^{2} a^{2}, f(\varphi), g(\varphi)$, and $h(\varphi)$ for both hemispheres as summer climatology (Fig. 2). While the first term $f(\varphi)$ is large close to the equator and decays toward the poles (Figs. 2c,h), the second term $g(\varphi)$ changes sign twice and exhibits a maximum at approximately $40^{\circ} \mathrm{N}\left(50^{\circ} \mathrm{S}\right)$. As $g(\varphi)$ inhibits the second-order derivative $d^{2} U / d \varphi^{2}$ this change of sign is primarily due to the changing inclination on the equatorward flank of $U$ (Figs. 2d,i). The third term $h(\varphi)$ is mostly independent of latitude with negligible contributions at the latitudes of interest $\left(30^{\circ}-70^{\circ} \mathrm{N}\right.$ and $\left.70^{\circ}-30^{\circ} \mathrm{S}\right)$ (Figs. 2e,j). Thus, in particular $g(\varphi)$ and therefore $d^{2} U / d \varphi^{2}$ is relevant for the formation of waveguides by creating a "bump" in $l^{2}$ and thereby determining the positions of the TPs [i.e., see criterion (i)]. Further, at the latitudes where waveguides form, the value of $l^{2}$ is to a great extent determined by $f(\varphi)$. Assuming that it is the shape of $g(\varphi)$ that creates a waveguide and that $g(\varphi) \approx 0$ near the TPs, then $f(\varphi)$ determines the range of zonal wavenumbers $k$ for which a waveguide forms.

Through this scaling analysis, we derive an expression for the relationship between $f(\varphi)$ and $k_{0}$, the climatological lowest wavenumber that can still be trapped. Under the assumption that at a TP, where $l^{2}=0$, the second term is also $g(\varphi) \approx 0$, and neglecting the third term $h(\varphi)$, Eq. (9) leads to

$$
k_{0}^{2}=f(\varphi)+1 \Rightarrow k_{0}=\sqrt{f(\varphi)+1} .
$$

Figures $2 b$ and $2 g$ show that a TP can usually be found in upper latitudes (i.e., $60^{\circ} \mathrm{S}$ and $60^{\circ} \mathrm{N}$ ). We conclude that it

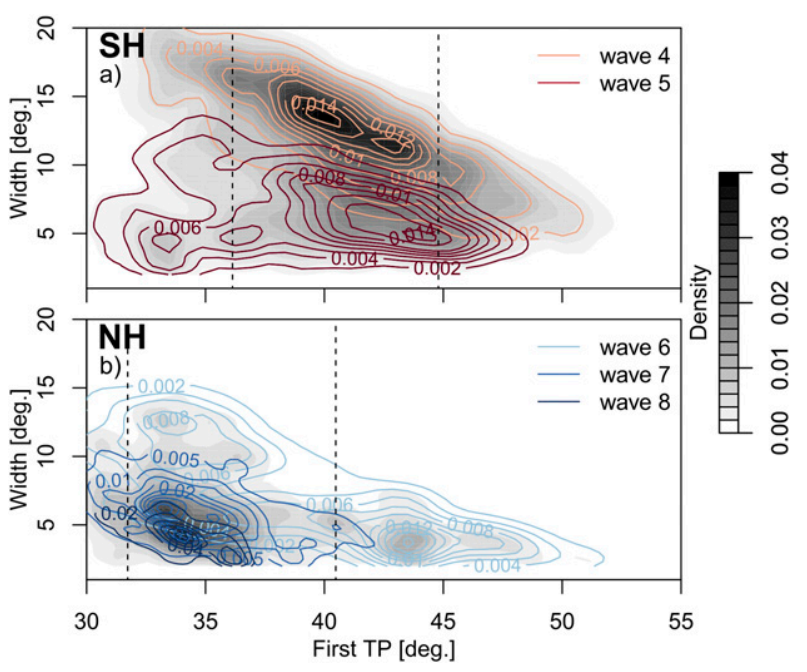

FIG. 3. Probability density plots of detected waveguide's first TP vs their widths for (a) SH for wavenumbers 4 and 5 (colored contours) and (b) $\mathrm{NH}$ for wavenumbers 6-8 and all respective wavenumbers combined (gray shading). An area of $\pm 1 \sigma$ relative to the mean TP position across all relevant wavenumbers of each hemisphere is marked by two vertical dashed black lines. The position of waveguides shifts south and widths decrease with increasing wavenumber. In (a), the waveguide positions in SH for wavenumbers 4 and 5 are predominantly distributed over a latitudinal range $37^{\circ}-45^{\circ} \mathrm{S}$. In (b), the waveguides for $\mathrm{NH}$ are mostly located at latitudes $32^{\circ}-40^{\circ} \mathrm{N}$ for wavenumbers 7 and 8 , whereas waveguides of wavenumber 6 are spread over latitudes $32^{\circ}-50^{\circ} \mathrm{N}$ exhibiting two maxima.

is the relatively rare presence of a turning point at the lower extratropics $\left(30^{\circ}-45^{\circ}\right)$ that is critical for a waveguide to form.

We determine the average position of this first TP as the point at which the climatological mean of $g(\varphi)$ changes sign $\left(41.1^{\circ} \mathrm{S}\right.$ and $\left.34.6^{\circ} \mathrm{N}\right)$, while the range of $\pm 1 \sigma$ (dashed lines) serves as an uncertainty measure (Figs. 2d,i). The average value of $f(\varphi)$ at these latitudes in combination with the range provided by the standard deviation $( \pm 1 \sigma)$ gives us an estimate for which zonal wavenumber waveguides can be expected in each hemisphere.

We obtain a value of $f_{\mathrm{SH}}(\varphi)=16.43$ and $f_{\mathrm{NH}}(\varphi)=$ 53.52 , which refer to values

$$
k_{0, \mathrm{NH}}=\sqrt{f_{\mathrm{NH}}+1}=7.4 \text { and } k_{0, \mathrm{SH}}=\sqrt{f_{\mathrm{SH}}+1}=4.2 .
$$

Considering $\pm 1 \sigma$ as the upper and lower boundary in $f(\varphi)$ we obtain ranges of $\Delta k_{0, \mathrm{SH}}=3.9-4.5$ and $\Delta k_{0, \mathrm{NH}}=5.8-8.9$ in the $\mathrm{SH}$ and $\mathrm{NH}$, respectively. Other approaches for estimating $\Delta k_{0}$ lead to similar ranges (Fig. S17 in the supplemental material). To investigate waveguide positions in both hemispheres, the $2 \mathrm{D}$ PDF of the first turning point and the waveguides widths (distance to second TP) for each wavenumber ( 3 and 4 for $\mathrm{SH}$ and $6-8$ for $\mathrm{NH}$ ) are plotted in Fig. 3. In the $\mathrm{NH}$, 
TABLE 1. Number of detected QRA conditions in days and events for wavenumbers 6-8 in the NH.

\begin{tabular}{lcccr}
\hline \hline NH condition/wave & Wavenumber 6 events/days & Wavenumber 7 events/days & Wavenumber 8 events/days & Total events/days \\
\hline (i) & $168 / 785$ & $152 / 873$ & $92 / 582$ & $412 / 2240$ \\
(i) + (ii) & $83 / 297$ & $151 / 796$ & $80 / 357$ & $314 / 1450$ \\
(i) + (ii) + AT & $71 / 249$ & $71 / 415$ & $24 / 113$ & $166 / 777$ \\
\hline
\end{tabular}

waveguides for wavenumbers $6-8$ show highest densities in the region $30^{\circ}-40^{\circ} \mathrm{N}$. Waveguides are widest for wavenumber 6 , with widths between $2.5^{\circ}$ and $15^{\circ}$ latitude. Waveguides for wavenumbers $7\left(2.5^{\circ}-10^{\circ}\right.$ latitude $)$ and 8 $\left(2.5^{\circ}-7.5^{\circ}\right.$ latitude) tend to be narrower (Fig. $\left.3 \mathrm{~b}\right)$. The probability density for wavenumber 6 shows two maxima at $35^{\circ}$ and $44^{\circ} \mathrm{N}$. The latter can be attributed to the polar jet, visible as a second peak in the zonal-mean zonal wind (Fig. 2f). Waveguides preferentially form at the (steep) subtropical jet, but can also form at the polar jet. As the polar jet is wavier its boundaries are generally much smoother in the zonal mean. Still, in some cases it is steep enough to reflect wavenumbers with comparatively long wavelengths (wavenumber 6) but not shorter wavelengths (e.g., wavenumbers 7 and 8). In the SH we observe waveguides in the region $40^{\circ}-55^{\circ} \mathrm{S}$ for wavenumbers 4 and 5 . Waveguides for wavenumber 4 have widths between $2.5^{\circ}$ and $20^{\circ}$ latitude in lower latitudes, while waveguides of wavenumber 5 exhibit widths between $2.5^{\circ}$ and $15^{\circ}$ latitude (Fig. 3a). Effectively, no waveguides are found for wavenumbers above 8 in the $\mathrm{NH}$ and above 5 in the SH.

\section{c. Characteristics of QRA events}

Following the results of above analysis we focus on wavenumbers $6-8$ in the $\mathrm{NH}$ and wavenumbers 4 and 5 in the SH. We apply QRA conditions (i), (i) + (ii), and (i) + (ii) + AT on the 1979-2015 summer months. Tables 1 and 2 show the number of days (in a 15-day running-mean picture) and events detected for the respective waves relevant in each hemisphere. The number of events detected for wavenumbers greater than 5 in the $\mathrm{SH}$ and greater than 8 in the $\mathrm{NH}$ was found to be insufficient for a statistical analysis (not shown). In the $\mathrm{NH}$ we detect 163 events for wavenumbers 6-8 in total, with an average duration of 4.6 days. Most events are detected for wavenumber 7 , which on average also show the longest duration ( 6 days). In the SH more and longer events are detected for wavenumbers 4 and 5. As the ratio of detected days between (i) and (i) + (ii) is about $2: 3$ in both hemispheres ( 0.65 for $\mathrm{NH}$ and 0.61 for $\mathrm{SH})$, the total numbers are much higher in the $\mathrm{SH}$. Here, waveguides are observed during approximately $70 \%$ of the analyzed period for wavenumber 4 , and $30 \%$ of the period for wavenumber 5 (the analyzed period being the total number of analyzed days, 3404 in the $\mathrm{NH}$ and 3240 in the $\mathrm{SH}$ ), whereas in the $\mathrm{NH}$ waveguides are observed for only $25 \%$ of the time for wavenumbers 6 and 7 . With an average duration of 6.9 days, $\mathrm{SH}$ events tend to be longer than $\mathrm{NH}$ events (4.9 days).

To analyze the characteristics of circulation patterns during QRA days we follow the approach introduced in Kornhuber et al. (2017). The climatological probability density functions (PDFs) of phase speed versus wave amplitude for wavenumbers 6-8 (NH; Fig. 4$)$ and 4 and 5 (SH; Fig. 5) for each hemisphere were determined. Then, the impact of each set of conditions, (i), (i) + (ii), and (i) + (ii) + AT, was investigated by determining the PDF anomalies. Consistent with the findings in Kornhuber et al. (2017) we observe an increasing probability of quasi-stationary high-amplitude waves for wavenumbers $6-8$ in the $\mathrm{NH}$ when stepwise including the conditions. A statistically significant increase in probability for regions with high-amplitude quasistationary waves $\left(c= \pm 2 \mathrm{~ms}^{-1}\right.$; range indicated with vertical black dashed lines) is observed for wavenumber 7 [(i)], wavenumbers 6 and 7 [(i) + (ii)], and wavenumbers 6 and $7[(\mathrm{i})+($ ii $)+$ AT]. Following the same procedure for wavenumbers 4 and 5 in the $\mathrm{SH}$, no significant difference for wavenumber 4 [(i)] is registered, while evident positive anomalies are observed for wave 5 number [(i)]. In the region of quasi-stationary waves these anomalies are statistically significant for wavenumber 5 . Days passing criterion (ii) show positive anomalies in the regions of high-amplitude quasi-stationary waves for wavenumbers 4 and 5 , with statistically significant shifts for both. Those anomalies become more pronounced after application of the AT.

For a better understanding of the specific shape of $U$ favoring QRA we analyze the 15-day running-mean $U$-wind profiles during resonance and nonresonance in the midlatitudes at $300 \mathrm{hPa}$. Profiles determined from QRA events and the climatological profiles, as well as their

TABLE 2. Number of detected QRA days and events for wavenumbers 4 and 5 in the $\mathrm{SH}$.

\begin{tabular}{lccc}
\hline $\begin{array}{c}\text { SH condition/ } \\
\text { wave }\end{array}$ & $\begin{array}{c}\text { Wavenumber 4 } \\
\text { events/days }\end{array}$ & $\begin{array}{c}\text { Wavenumber } 5 \\
\text { events/days }\end{array}$ & $\begin{array}{c}\text { Total } \\
\text { events/days }\end{array}$ \\
\hline (i) & $209 / 2313$ & $151 / 1015$ & $360 / 3328$ \\
(i) + (ii) & $217 / 1471$ & $129 / 572$ & $346 / 2043$ \\
(i) + (ii) + AT & $157 / 1263$ & $89 / 432$ & $246 / 1695$ \\
\hline
\end{tabular}


wave 6
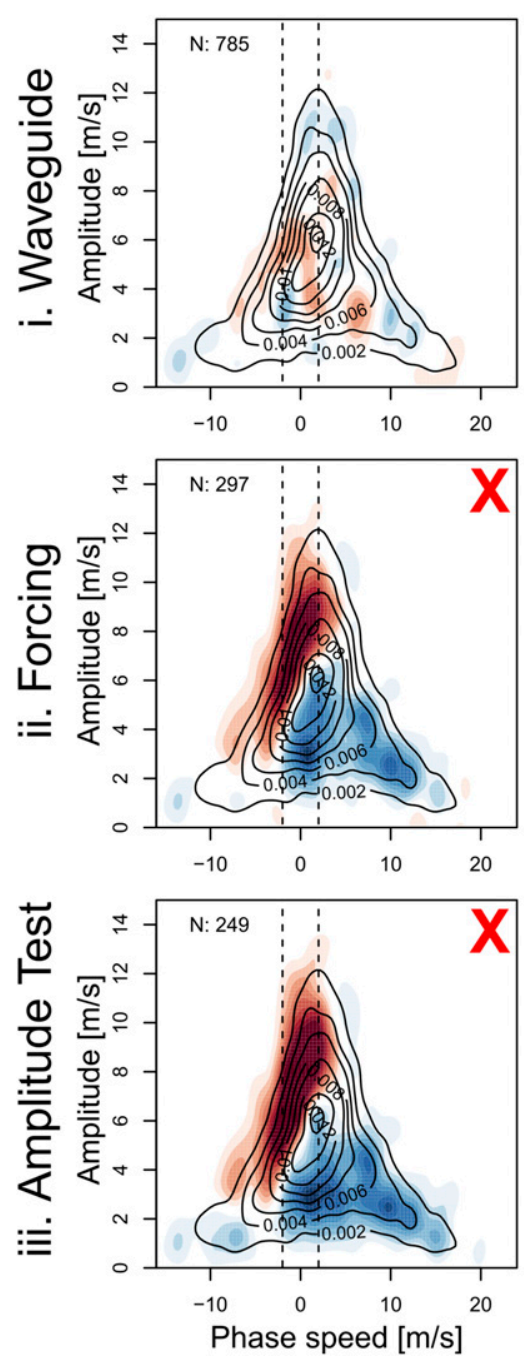

wave 7
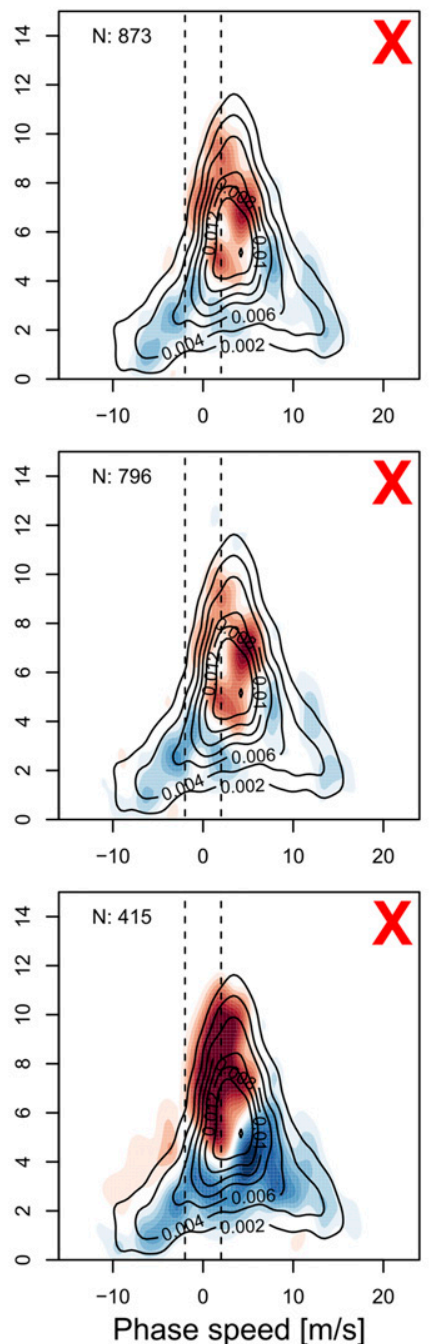

wave 8
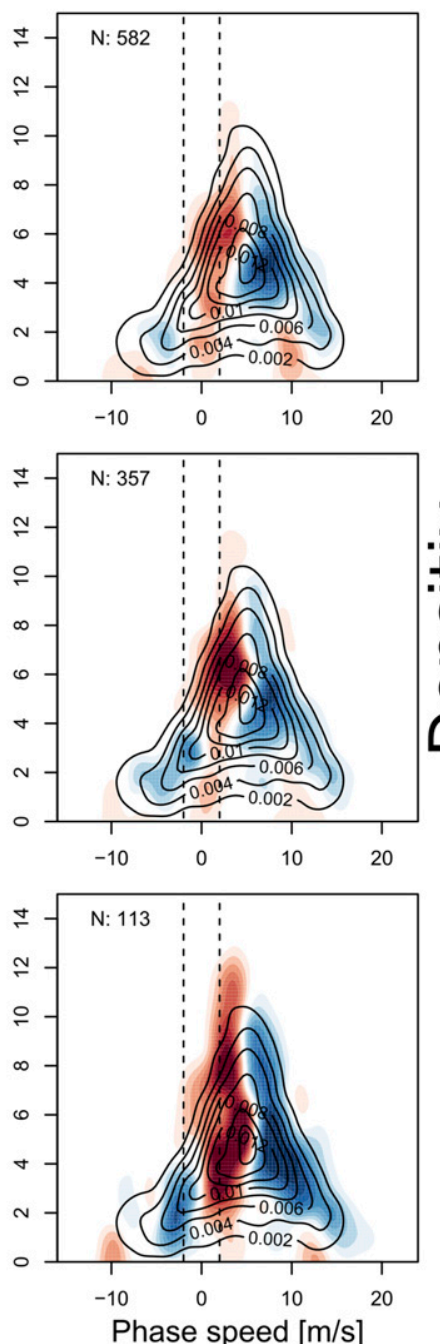

FIG. 4. Climatologies of the probability density distributions of wave speed vs wave amplitude of detected QRA days in the NH for wavenumbers 6-8 (black contours) and anomalies after applying QRA conditions (top) (i), (middle) (i) + (ii), and (bottom) (i) + (ii) + AT as blue-red shading. The area of stationary waves $|c|= \pm 2 \mathrm{~m} \mathrm{~s}^{-1}$ is depicted by dashed black vertical lines. Statistical significance for those waves is indicated with a red cross in the top-right corner of each panel. The sample size $N$ for each distribution is given in the topleft corner.

first-order derivatives, are shown for wavenumbers 4 and 5 in the $\mathrm{SH}$ and wavenumbers $6-8$ in the $\mathrm{NH}$ in Fig. 6. In both hemispheres QRA is related to stronger and steeper jets in the zonal average. In the $\mathrm{SH}$ the jet exhibits an equatorward shift of about $5^{\circ}$ latitude for wavenumber 5 compared to the DJF climatology (Fig. 6b) and a stronger gradient is observed at the equatorward flank, while for wavenumber 4 the differences in jet strength are small. In the $\mathrm{NH}$, higher wind speeds are observed in the zonal average during QRA (Figs. 6c,d) for wavenumbers 6 and 7. Steeper gradients are observed on both flanks leading to a stronger, narrower jet. For wavenumber 8 the jet is weaker on average but slightly shifted equatorward (Fig. 6e). A second peak in $U$ emerges at higher latitudes $\left(70^{\circ} \mathrm{N}\right)$ during QRA of wavenumbers 6-8.

\section{d. QRA phase positions}

The waves' phase position determines the hemispheric distribution of north-south wind speeds and thus the regions that are potentially affected by stationary weather patterns. We compare the distributions of phase positions based on 15-day running-mean meridional wind fields for each wavenumber during QRA and nonQRA episodes in order to investigate if QRA favors certain phase positions over others. Figure 7 shows the 

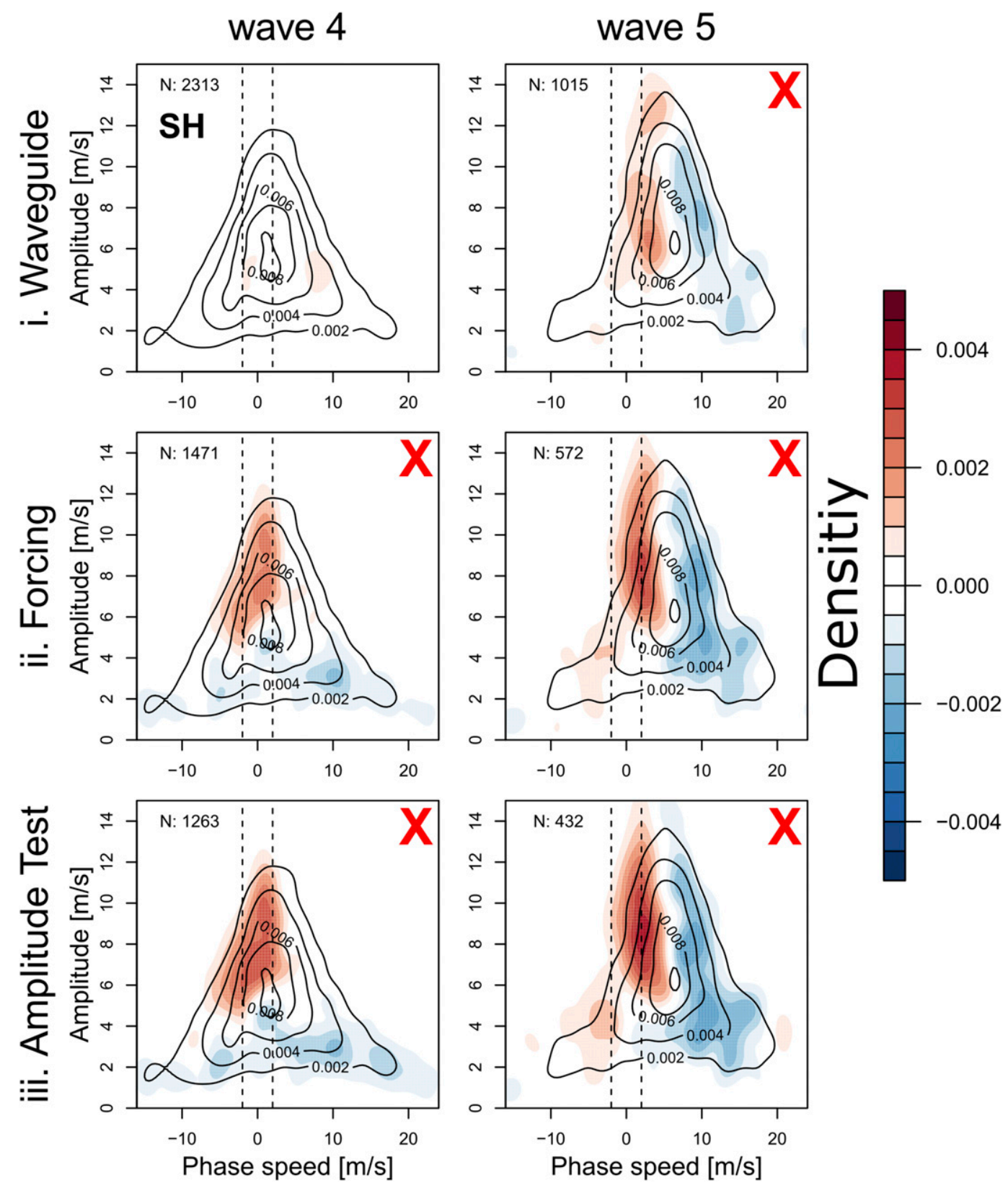

FIG. 5. As in Fig. 4, but in the SH for wavenumbers 4 and 5.

probability density distributions of phases of wavenumbers 4 and 5 in the SH (Figs. 7a,b) and wavenumbers 6-8 in the NH (Figs. 7c-e) for QRA events (colored line), and climatology (black line). The $25 \%-75 \%(5 \%-95 \%)$ uncertainty ranges are shown as dark (light) gray shading. The preferred phases are marked by a red vertical line, while those phases are margined by red dashed lines. The uncertainty ranges of the preferred phase positions (red dashed lines) were determined by a bootstrapping method. Sampling from the list of detected events we generated 10000 new sets (with resampling) with the same total number of events as found in the original set. Preferred phase positions were determined from each of the resulting distributions and from the ensembles a $5 \%-95 \%$ uncertainty range was determined. A bootstrapping approach was also applied to test the statistical significance of the QRA phase distributions. Subsets (10000) from the whole set of available summer time steps were sampled, while mimicking the duration distribution and number of the detected QRA events for each wave. This was done to conserve the autocorrelation. 

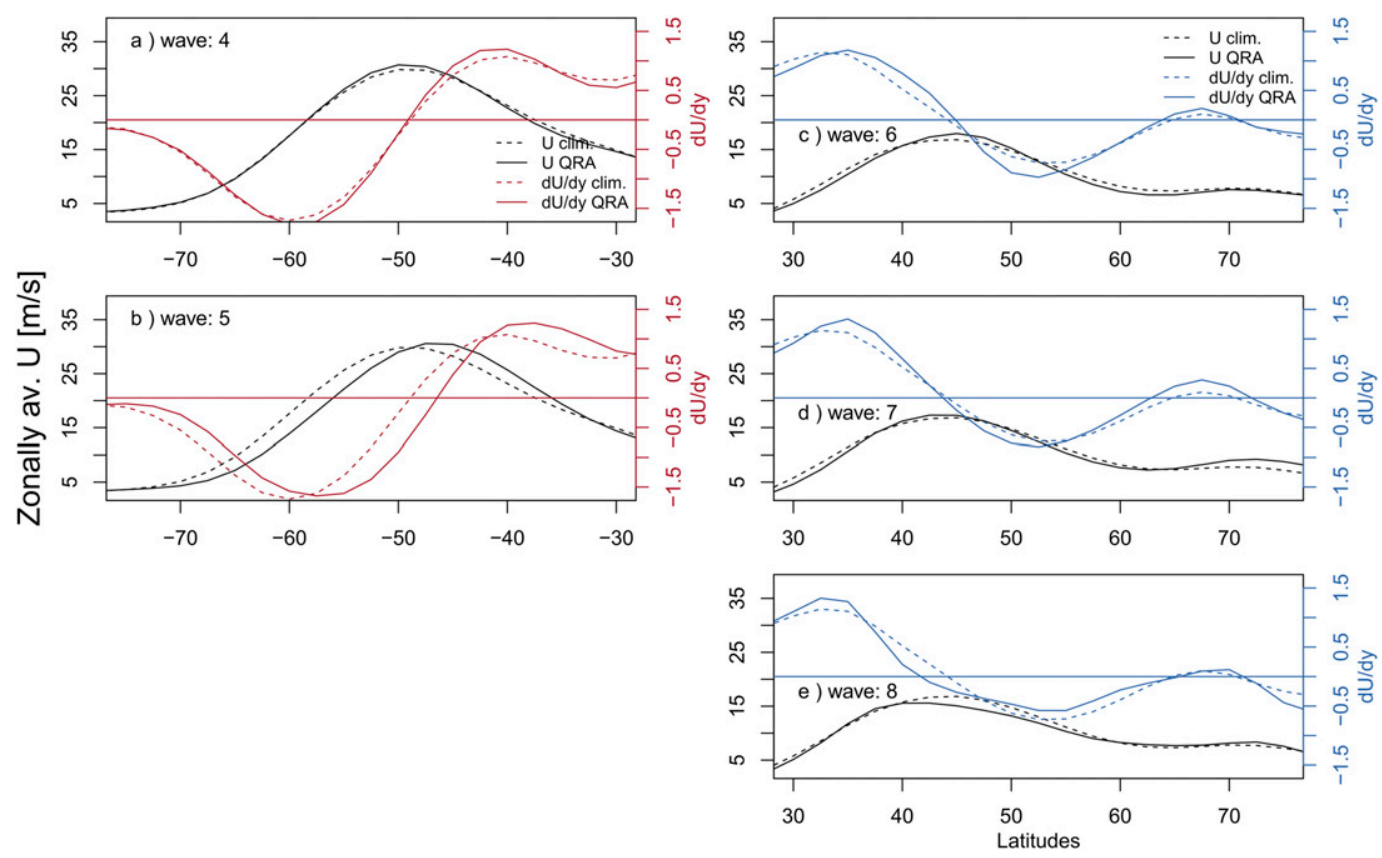

FIG. 6. Profiles of zonally averaged $U$ comparing summer climatology (dashed black line) and QRA events (solid black line) of wavenumbers (a),(b) 4 and 5 for SH and (c)-(e) 6-8 for NH. First derivatives are shown in red (blue) in the $\mathrm{SH}(\mathrm{NH})$. A strong jet with a sharp equatorward flank is associated with QRA in both hemispheres.

Phase positions are given in Earth longitudes relative to the date line $\left(180^{\circ}\right)$. In each panel the sample size $N$ is provided at the top-right. In the $\mathrm{SH}$ a bimodal behavior is observed for wavenumbers 4 and 5 (Figs. 7a,b). The distribution of wavenumber 4 shows phase maxima at $p_{1}=16^{\circ}$ and $p_{2}=-11^{\circ}$ during QRA (Fig. 7a), while the climatology is distributed broadly over the entire spectrum with a slight preference for phases around $20^{\circ}$. During non-QRA this peak decreases. The climatological distribution for wavenumber 5 has minor phase peaks at $19^{\circ}$ and $-29^{\circ}$ but is almost uniformly distributed over the entire longitudinal range. During QRA two preferred phase positions are detected for wavenumber 5 , one at $p_{1}=29^{\circ}$ and the other at $p_{2}=-19^{\circ}$ (Fig. 7b). All preferred phase positions found for wavenumbers 4 and 5 in the SH are outside the $95 \%$ uncertainty range and can thus be considered statistically significant.

NH phase probability density distributions of wavenumbers 6-8 are displayed in Figs. 7c-e. In the $\mathrm{NH}$ only wave 6 shows a bimodal behavior during QRA with two peaks at $p_{1}=10^{\circ}$ and $p_{2}=-20^{\circ}$ (Fig. 7c). Here, the climatological values do not show a clear preference with a slight maximum close to zero. The preferred positions for waveumber 6 are not statistically significant at $95 \%$ confidence, although they fall outside the $25 \%-$ $75 \%$ uncertainty range. Wavenumbers 7 and 8 exhibit a single maximum in the phase density distribution during
QRA episodes. During QRA a pronounced peak is observed for wavenumber 7 at $p_{1}=-9^{\circ}$. This value falls together with the dominant seasonal phase position but shows a distinctively stronger peak (Fig. 7d). Wavenumber 8 shows a peak in climatology between $0^{\circ}$ and $20^{\circ}$. During QRA a single peak at $p_{1}=5^{\circ}$ is observed (Fig. 7e). Both peaks are found to be significantly different from the climatological distribution.

\section{e. Composite v-wind and temperature anomaly maps of preferred phase positions}

To visualize the importance of particular phase positions for regional weather patterns, we create composites of $v$-wind and near-surface temperature for those preferred phase positions. QRA time steps with the observed phase position within the uncertainty range of the preferred phase position $p$ (given by the red dashed vertical lines in Fig. 7) are used for the composites. Composite fields are shown in Fig. 8 for peaks $p_{1}$ and $p_{2}$ of wavenumbers 4 and 5 in the $\mathrm{SH}$ and in Fig. 9 for peaks $p_{1}$ and $p_{2}$ of wavenumber 6 and $p_{1}$ for wavenumbers 7 and 8 in the NH. The sample size $N$ and the longitudinal preferred phase position are provided on the left side of each panel and significant grid points are marked with black dots. Composite $v$-wind maps for non-QRA episodes and $\mathrm{SH} / \mathrm{NH}$ summer climatology are provided in Fig. S16 of the supplemental material. 

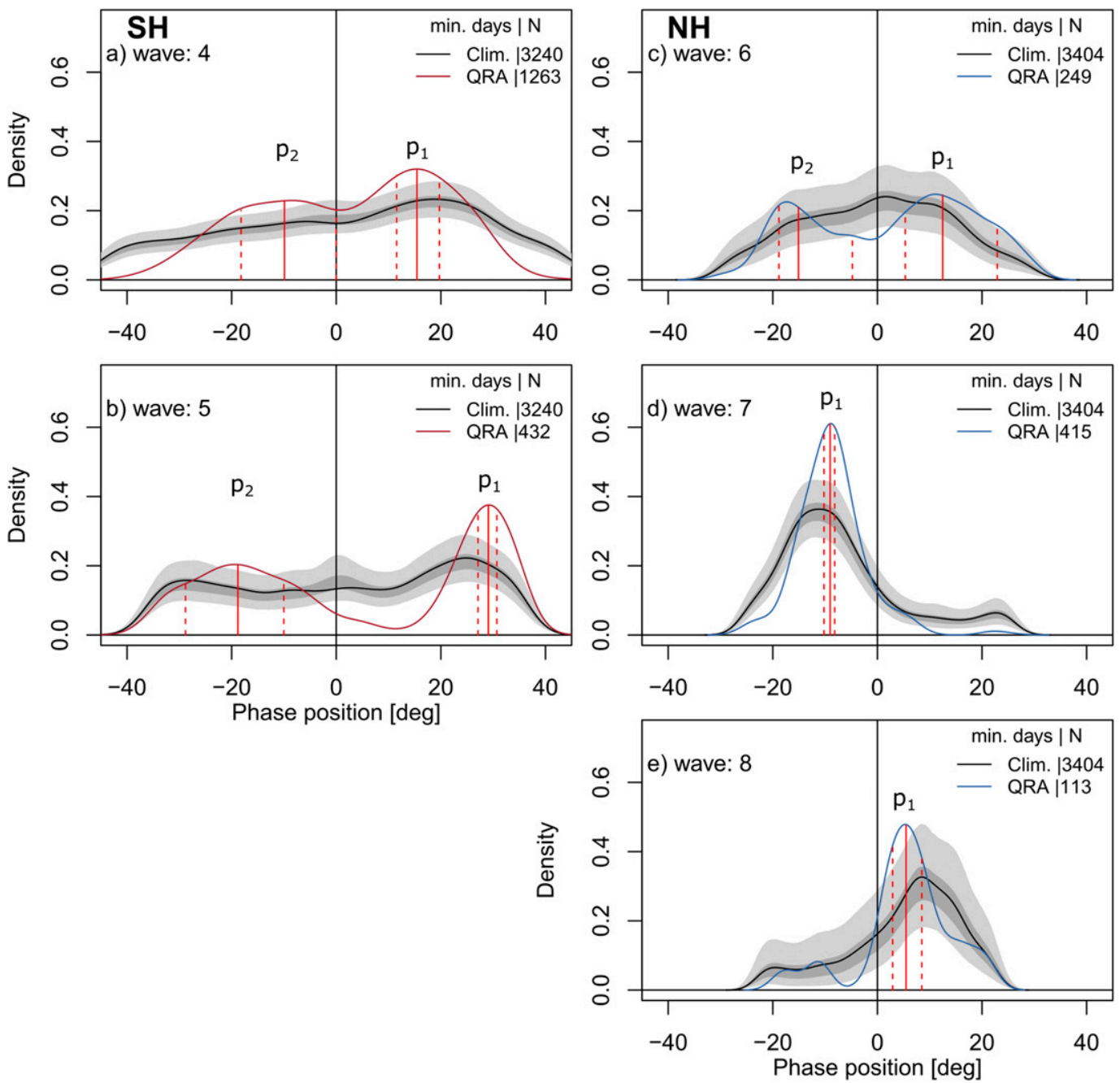

FIG. 7. Probability density distributions of phase positions for wavenumbers (a),(b) 4 and 5 in SH and (c)-(e) 6-8 in NH for summer climatology (black curve), during detected QRA events (red and blue curves). Maxima occurring in the QRA phase distributions are marked with vertical red lines and $p_{1}$ and $p_{2}$ in the order of their magnitude. Red vertical dashed lines mark the $5 \%$ and $95 \%$ quantile determined by a bootstrapping method. The dark (light) gray areas mark the range defined by the $25 \%-65 \%(5 \%-95 \%)$ quantile around the climatological phase distribution. Peaks with a probability distribution beyond the $95 \%$ range are considered to be statistically significant. Except for $p_{1}$ and $p_{2}$ of wavenumber 6 all identified peaks are statistically significant.

For the peaks for wavenumbers 4 and 5 in the $\mathrm{SH}$, the meridional wind fields depict alternating northward and southward wind patterns that correspond to the respective wavenumber (Fig. 8). The largest differences in the position of $v$ winds between wavenumbers are observed in the Western Hemisphere $\left(180^{\circ}-0^{\circ}\right)$, while the positions of the strongest north-south wind speeds are shifted by a few degrees only. The ridge pattern over eastern Australia observed for $p_{1}$ and $p_{2}$ of wavenumber 4 and $p_{1}$ of wavenumber 5 is a climatological feature termed the summertime subtropical ridge and is also observed during non-QRA days (Fig. S16). In the SH, positive temperatures are found between southward and northward flows (as seen from east to west), while negative temperature anomalies tend to be situated between pairs of northward and southward flows. Temperature anomalies over $1^{\circ} \mathrm{C}$ are mostly significant. Ocean areas exhibit weaker temperature anomalies are due to higher heat capacity of water, a mixed ocean layer typically $100 \mathrm{~m}$ deep, and the possibility of heat transport to even deeper ocean levels. The strongest temperature anomalies over southern Australia are observed for $p_{1}$ of wavenumber 5 (Fig. 8c). This seems consistent with Parker et al. (2014) and Pezza et al. (2012), who identified a wavenumber-5 pattern to be important for southern Australian heat extremes. 


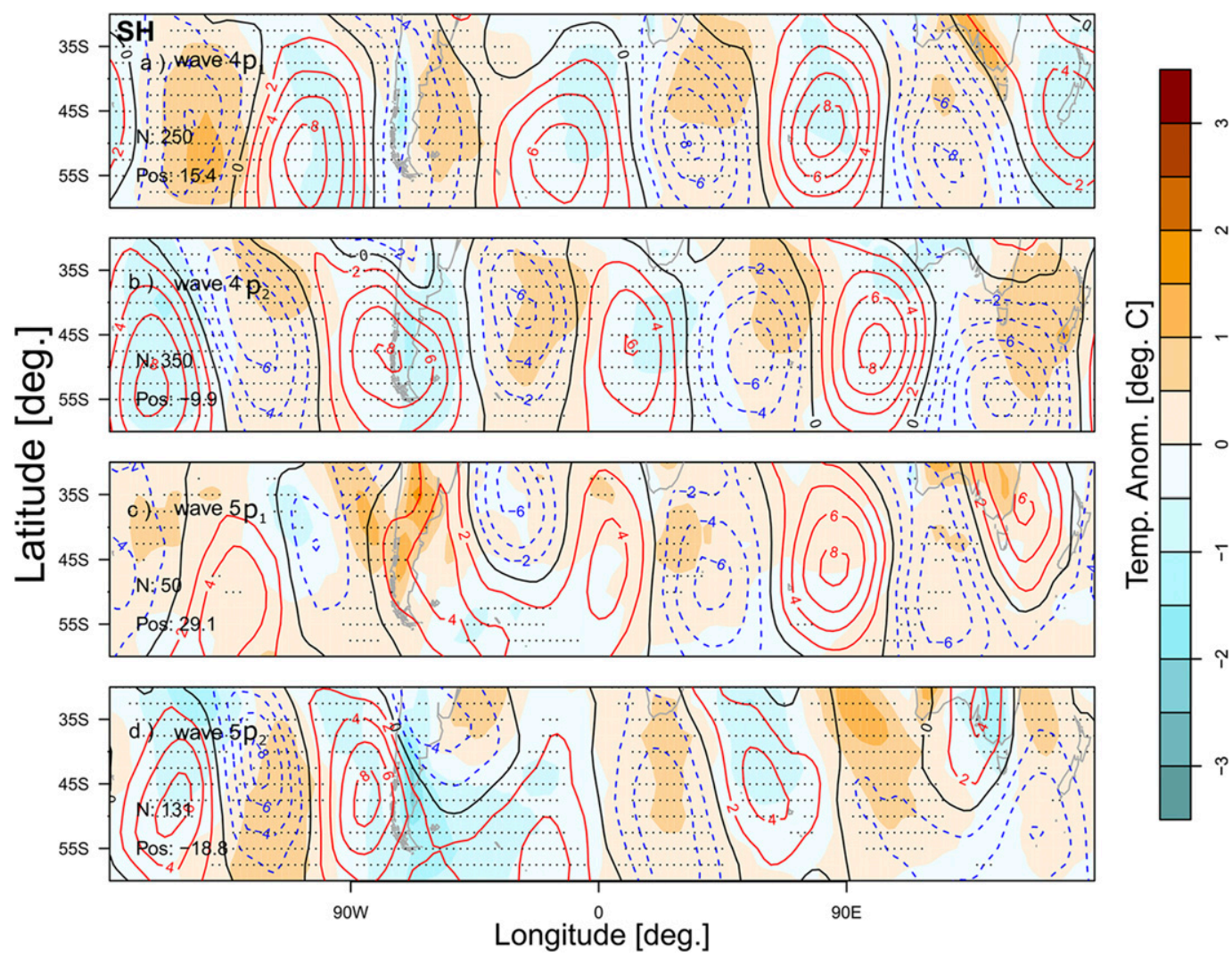

FIG. 8. Composite $v$-wind (contours) and temperature anomaly field (color shading; ${ }^{\circ} \mathrm{C}$ ) associated with the preferred phases (a),(c) $p_{1}$ and (b),(d) $p_{2}$ during QRA days for wavenumbers (a),(b) 4 and (c),(d) 5 in the SH. Positive wind speeds are shown as red contours and negative wind speeds are shown as dashed blue contours, with an interval of $2 \mathrm{~m} \mathrm{~s}^{-1}$ between contours. The zero wind line is shown as a black solid contour. Black dots mark grid points with significant temperature anomalies compared to DJF climatology determined by the Student's $t$ test $(p>0.05)$.

Composite $v$-wind fields and temperature anomaly fields were generated for wavenumbers $6-8$ in the $\mathrm{NH}$ likewise and are shown in Fig. 9 for $p_{1}$ and $p_{2}$ respectively. The $v$-wind fields of wavenumbers 6 and 7 for $p_{1}$ exhibit a distinct number of pairs of north-south flows respectively matching the analyzed wavenumber (Fig. 9). The meridional wind field of wavenumber 8 , however, resembles a wavenumber-7 pattern (Fig. 9d). Strongest differences are observed over Asia $\left(90^{\circ} \mathrm{E}-\right.$ $180^{\circ}$ ), where wavenumbers 7 and 8 exhibit an additional ridge-trough swing compared to wavenumber 6 . Shared patterns consist of a northward directed flow at the U.S. west coast, slightly shifted inland with increasing wavenumber and a ridge over western Europe. Temperature anomalies in the midlatitudes mirror the composite $v$-wind fields and are mostly found to be statistically significant above a level of $1^{\circ} \mathrm{C}$ compared to JJA climatology. Strong positive anomalies are found predominantly where meridional winds are low (zero wind line: black solid lines in Fig. 9). Compared to the
$\mathrm{SH}$, surface temperature anomalies in the $\mathrm{NH}$ are larger. This can be explained by the larger land area here. Anomalies over oceans are similar in magnitude between hemispheres. Temperature anomalies are found to be strongest in the Western Hemisphere over the west coast of North America and western-central Europe for $p_{1}$ of wavenumbers 6-8. In Fig. 9b a $v$-wind map derived from the secondary peak detected in wavenumber-6 probability density distribution of phase position is shown. North-south wind patterns are shifted by about half a wavelength compared to the wind field shown in Fig. 9a. Here, the ridges are located over central United States and eastern Europe while the position of north-south wind fields remain unchanged over Asia. The temperature anomalies are shifted correspondingly, with the most pronounced positive anomalies over central Eurasia. The secondary peak of wavenumber 6 relates to a $v$-wind field with an eastward-shifted north-south flow, constituting a pattern observed during the 2010 heat wave in Russia. For 


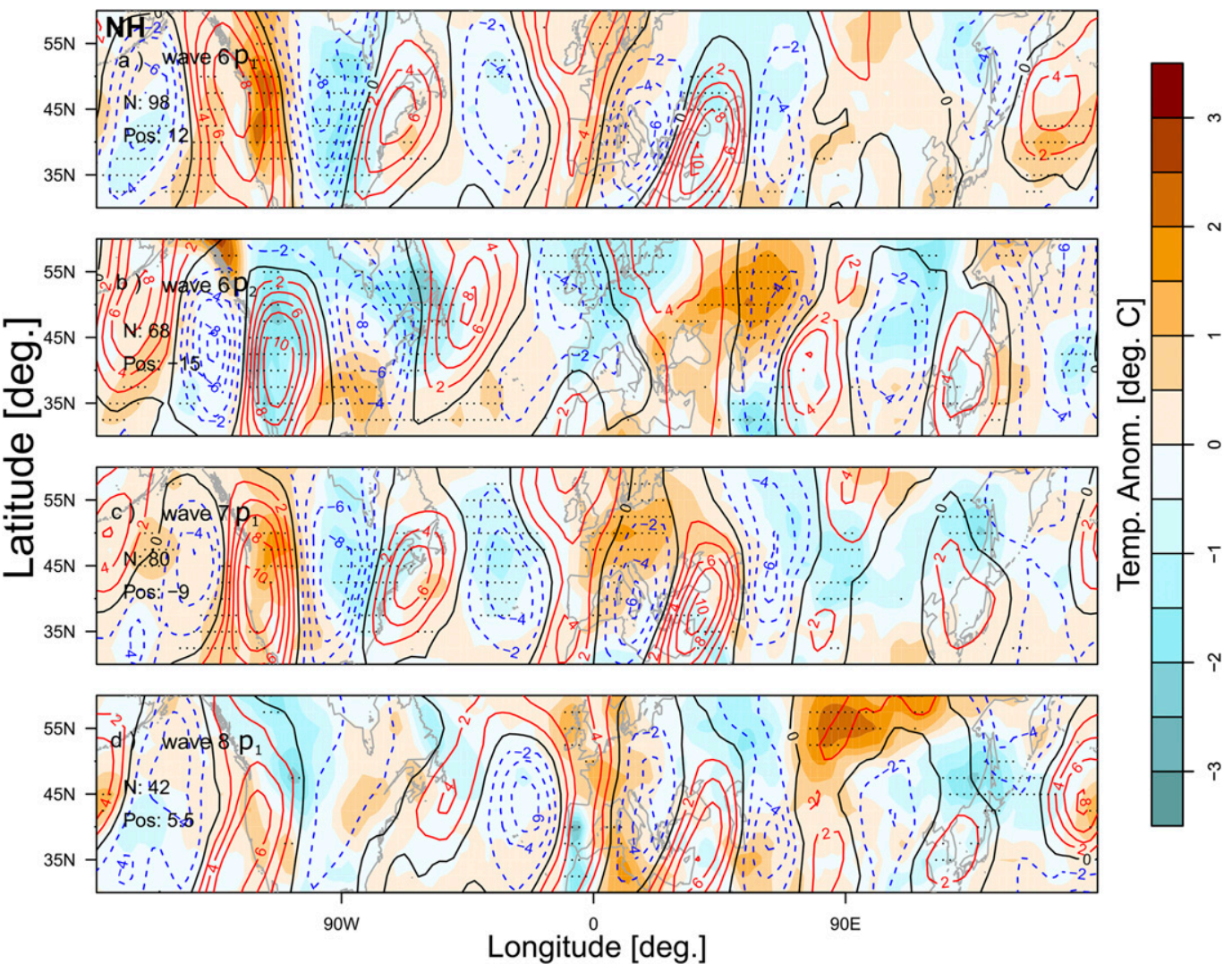

FIG. 9. Composite $v$-wind (contours) and temperature anomaly field (color shading; ${ }^{\circ} \mathrm{C}$ ) of preferred phases (a),(c),(d) $p_{1}$ and (b) $p_{2}$ during QRA days for wavenumbers (a),(b) 6, (c) 7, and (d) 8 in the NH. Positive wind speeds are shown as red contours and negative wind speeds are shown as dashed blue contours, with an interval of $2 \mathrm{~m} \mathrm{~s}^{-1}$ between contours. The zero wind line is shown as a black solid contour. Black dots mark grid points with significant temperature anomalies compared to JJA climatology determined by the Student's $t$ test $(p>0.05)$.

comparison, Fig. 10 shows the meridional wind patterns and temperature anomalies during the European heat wave of 2003 (Fig. 10a) and the Russian heat wave of 2010 (Fig. 10b), which were identified as QRA events of wavenumbers 6 and 7 and wavenumber 6 , respectively (Petoukhov et al. 2013; Kornhuber et al. 2017). Both climatological and non-QRA maps (Figs. S16c,d) resemble a wavenumber-7 pattern. Both exhibit much weaker wind speeds (i.e., reduced wave amplitudes) than during QRA episodes.

\section{Discussion and conclusions}

We present evidence that QRA can act as an amplifying mechanism for planetary waves in both the Northern and Southern Hemisphere. Differences in the climatological magnitude and shape of the zonalmean zonal wind between hemispheres result in a different set of wavenumbers being susceptible to QRA in each hemisphere. In the NH QRA is important for wavenumbers $6-8$, consistent with previous studies (Petoukhov et al. 2016; Kornhuber et al. 2017), and in the SH it is important for wavenumbers 4 and 5. The stronger jets in the SH lead to a lower stationary wavenumber ( 4 and 5) compared to the weaker jets in the NH (wavenumbers 6-8) (Fig. 1). This also follows from Eq. (1), which shows that stronger jets (entering the equation as zonal average $U$ in the denominator) decrease the value of $K_{s}^{2}$, resulting in lower values of the zonal wavenumber $k$ in the SH itself. This is finding is also consistent with theoretical scaling analyses resulting in decreased baroclinicity and zonal winds as a first-order outcome of Arctic amplification (Hoskins and Woollings 2015). During QRA, the quasi-stationary components of wavenumbers 4 and 5 have significantly magnified amplitudes in the $\mathrm{SH}$ (Fig. 5). The shape and magnitude of the zonally averaged zonal mean wind in the $\mathrm{SH}$ summertime circulation is such that it creates a waveguide for wavenumber 4 during approximately two-thirds of the 


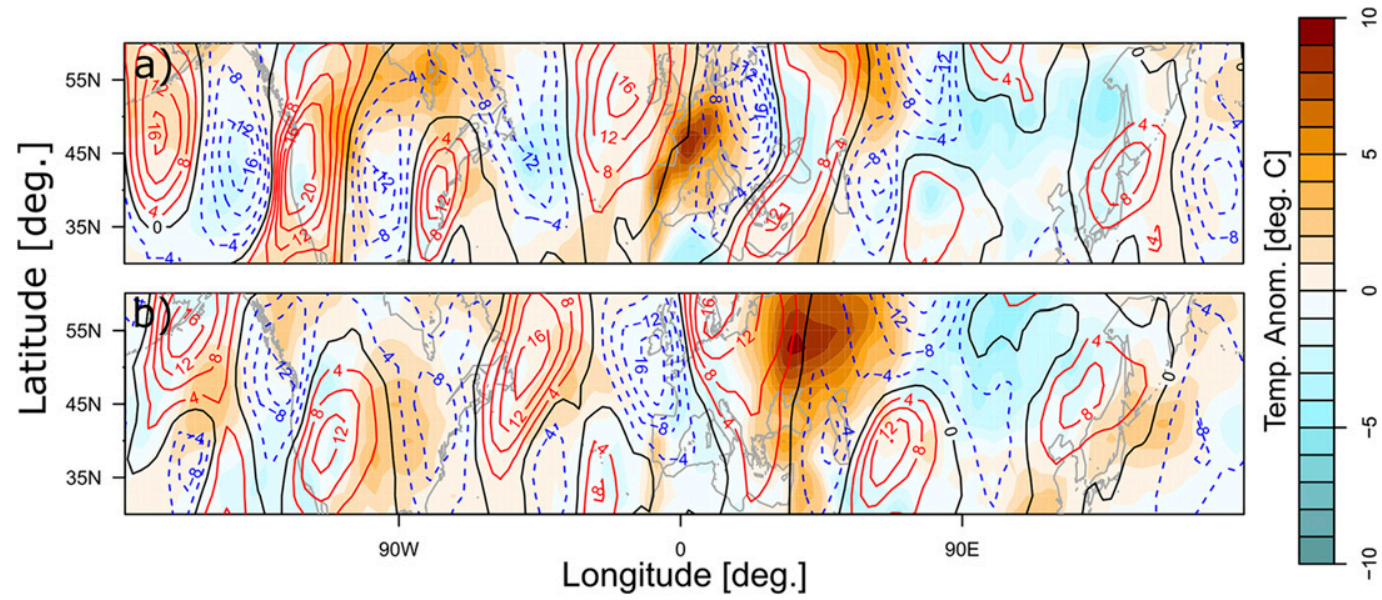

FIG. 10. Meridional wind (contours) and temperature anomalies (color shading; ${ }^{\circ} \mathrm{C}$ ) during (a) the European heat wave of 2003 and (b) the Russian heat wave of 2010. Positive wind speeds are shown as red contours and negative wind speeds are shown as dashed blue contours, with an interval of $4 \mathrm{~m} \mathrm{~s}^{-1}$ between contours. The zero wind line is shown as a black solid contour.

investigated period. An equatorward turning point, the crucial one for waveguide formation, is a climatological feature for wavenumber 4 (Fig. 2b). For wavenumbers above 5 , however, no waveguides were found. This wavenumber range susceptible for waveguides in the $\mathrm{SH}$ compares well with those identified using clustering methods (O'Kane et al. 2016) and experiments using a barotropic channel model (Manola et al. 2013). QRA episodes in the SH are longer ( $\sim 7$ days) than in the NH ( $\sim 5$ days) (Tables 1 and 2), which is attributed to an increased relative variability of $U$ at the waveguide-relevant latitudes (Fig. S19 in the supplemental material). In agreement with earlier studies (Ambrizzi et al. 1995; Branstator 1983; Manola et al. 2013) we find that fast and narrow zonal winds act as waveguides (Fig. 6). Kornhuber et al. (2017) showed that the NH double-jet structure is strongly linked to QRA as this is associated with a steep subtropical jet. Double-jet situations are not important features of QRA in the SH (Figs. 6a,b). Instead, the single zonal mean jet is stronger, with a steeper latitudinal profile, and more shifted equatorward during QRA episodes compared to nonresonance days.

QRA relevant waves have specific preferred longitudinal phase positions, which differ significantly from the climatological distribution for all waves except for wavenumber 6 in the NH. A bimodal behavior of the phase positions of wavenumbers 4 and 5 during QRA in the $\mathrm{SH}$ and for wavenumber 6 in the $\mathrm{NH}$ is found, while wavenumbers 7 and 8 exhibit a single preferred phase (Fig. 7). Composite $v$-wind and temperature anomaly maps based on the identified phase positions reveal those regions in the midlatitudes likely to experience either cold or warm anomalies during QRA events. The $v$-wind patterns of the primary preferred phase $p_{1}$ of wavenumbers 4 and 5 lead to positive temperature anomalies over large parts of Australia (southerlies over western Australia and northerlies over the east (Parker et al. 2014; Pezza et al. 2012) (Fig. 8). In the NH the primary preferred phases $p_{1}$ for wavenumbers 6-8 share a pronounced north-south flow over the western United States, western Europe, and western central Asia, causing significant positive temperature anomalies in these regions (Fig. 9). Similar wave train patterns were observed during the European heat wave of 2003 (Kornhuber et al. 2017) (Fig. 10a). The secondary preferred phase $p_{2}$ of wavenumber 6 corresponds to a $v$-wind pattern observed during the Moscow heat wave of 2010 (Fig. 10b) (Petoukhov et al. 2013).

The existence and the position of preferred phases can likely be explained by the location of prominent orographic structures (e.g., the Rocky Mountains or Ural Mountains in the NH or the Andes in the SH) and the position of continents and oceans resulting in surface temperature differences. In the $\mathrm{NH}$, locations of the zero wind line (black solid line) tend to be situated at the west coasts of the continents (California, Europe) resulting in northward winds offshore and southward winds onshore, or vice versa. Thus, high temperature anomalies over land in the vicinity of the coast line in summer impose a preferred direction on the $v$ winds, due to thermal wind relation. This would explain why the phase positions leading to warm anomalies over the western coasts are the ones that are most preferred for all wavenumbers. 
In the $\mathrm{SH} v$ winds seem to be regulated to some extent by the strongest, orographic-type sources, like the Andes. It forces the flow to make either a northward or a southward swing at its upwind side, resulting in a bimodal phase distribution of resonant wavenumbers 4 and 5. Other active barotropic sources arranging the preferred positions of these wavenumbers are Australia, New Zealand, South Africa, and the central part of the southern Indian Ocean. As there are fewer continents in the $\mathrm{SH}$, the difference in land ocean temperature differences is generally smaller in the $\mathrm{SH}$ (Fig. 8). We note that it is the lower wavenumbers ( 4 and 5 in the $\mathrm{SH}$ and 6 in the $\mathrm{NH}$ ) that show a bimodal behavior, while for higher wavenumbers ( 7 and 8 in the $\mathrm{NH}$ ) this behavior is less pronounced.

The theoretical framework developed by Petoukhov et al. (2013) for the NH summer circulation explaining the occurrence of high-amplitude waves of high wavenumbers (6-8) is also valid for SH summer circulation but for a different set of wavenumbers (4 and 5). When waves are trapped by a waveguide and amplified by resonance given a small forcing, a circumglobal wave of hemisphere specific wavenumbers can be generated. The results add to the body of evidence of the existence of multiple flow equilibria in the atmospheric circulation as first suggested by Charney and De Vore (1979) and further investigated by, among others, Yang et al. (1997), Zidikheri et al. (2007), and Lutsko and Held (2016). A particular difference of the QRA mechanism discussed here, however, is that it acts on those wavenumbers that were before generally considered to occur mostly as transient waves in their respective hemisphere (Dell'Aquila et al. 2005).

Acknowledgments. The authors thank Terence O'Kane and an anomymous reviewer for their valuable comments, which much improved this manuscript, and Claire Fyson and Jascha Lehmann for support in final editing of the manuscript. The authors thank the ERA-Interim project at the ECMWF for making their data available. We also gratefully acknowledge the European Regional Development Fund (ERDF), the German Federal Ministry of Education and Research (BMBF) and the Land Brandenburg for supporting this project by providing resources on the high performance computer system at the Potsdam Institute for Climate Impact Research. Kai Kornhuber thanks the German Academic Exchange Service (DAAD) for the financial support and the German-Australian Climate and Energy College for their hospitality and support during a research stay at the University of Melbourne. The presented work was supported by the BMBF (Grant 01LN1304A).

\section{REFERENCES}

Ambrizzi, T., B. J. Hoskins, and H.-H. Hsu, 1995: Rossby wave propagation and teleconnection patterns in the austral winter. J. Atmos. Sci., 52, 3661-3672, doi:10.1175/ 1520-0469(1995)052<3661:RWPATP>2.0.CO;2.

Black, E., M. Blackburn, G. Harrison, B. Hoskins, and J. Methven, 2004: Factors contributing to the summer 2003 European heatwave. Weather, 59, 217-223, doi:10.1256/wea.74.04.

Branstator, G., 1983: Horizontal energy propagation in a barotropic atmosphere with meridional and zonal structure. J. Atmos. Sci., 40, 1689-1708, doi:10.1175/1520-0469(1983)040<1689: HEPIAB $>2.0 . \mathrm{CO} ; 2$.

- 2002: Circumglobal teleconnections, the jet stream waveguide, and the North Atlantic Oscillation. J. Climate, 15, 1893-1910, doi:10.1175/1520-0442(2002)015<1893:CTTJSW>2.0.CO;2.

Charney, J. G., and A. Eliassen, 1949: A numerical method for predicting the perturbations of the middle latitude westerlies. Tellus, 1, 38-54, doi:10.3402/tellusa.v1i2.8500.

- and J. De Vore, 1979: Multiple flow equilibria in the atmosphere and blocking. J. Atmos. Sci., 36, 1205-1216, doi:10.1175/ 1520-0469(1979)036<1205:MFEITA > 2.0.CO;2.

Coumou, D., V. Petoukhov, S. Rahmstorf, S. Petri, and H. J. Schellnhuber, 2014: Quasi-resonant circulation regimes and hemispheric synchronization of extreme weather in boreal summer. Proc. Natl. Acad. Sci. USA, 111, 12331-12336, doi:10.1073/pnas.1412797111.

Dee, D. P., and Coauthors, 2011: The ERA-Interim reanalysis: Configuration and performance of the data assimilation system. Quart. J. Roy. Meteor. Soc., 137, 553-597, doi:10.1002/qj.828.

Dell'Aquila, A., V. Lucarini, P. M. Ruti, and S. Calmanti, 2005: Hayashi spectra of the Northern Hemisphere mid-latitude atmospheric variability in the NCEP-NCAR and ECMWF reanalyses. Climate Dyn., 25, 639-652, doi:10.1007/s00382-005-0048-x.

Dingle, R. B., 1973: Asymptotic Expansions: Their Derivation and Interpretation. Academic Press, $521 \mathrm{pp}$.

Dionne, B. P., H. L. Mitchell, and J. Derome, 1988: On the resonance of an external planetary wave and its interaction with the mean zonal flow. Tellus, 40A, 177-187, doi:10.1111/ j.1600-0870.1988.tb00340.x.

Frederiksen, J. S., and P. J. Webster, 1988: Alternative theories of atmospheric teleconnections and low-frequency fluctuations. Rev. Geophys., 26, 459-494, doi:10.1029/RG026i003p00459.

Hastings, D., and P. Dunbar, Eds., 1999: Global land one-kilometer base elevation (GLOBE) digital elevation model: Documentation, version 1.0, documentation volume 1.0. Key to Geophysical Records Documentation (KGRD) 34, NOAA, NGDC, 139 pp.

Haurwitz, B., 1940a: The motion of atmospheric disturbances. J. Mar. Res., 3, 35-50.

, 1940b: The motion of atmospheric disturbances on the spherical Earth. J. Mar. Res., 3, 254-267.

Held, I. M., 1983: Stationary and quasi-stationary eddies in the extratropical troposphere: Theory. Large-Scale Dynamical Processes in the Atmosphere, B. J. Hoskins and R. P. Pearce, Eds., Academic Press, 127-168.

— R. L. Panetta, and T. H. Pierrehumbert, 1985: Stationary external Rossby waves in vertical shear. J. Atmos. Sci., 42, 865-883, doi:10.1175/1520-0469(1985)042<0865:SERWIV>2.0.CO;2.

Hoskins, B. J., and D. J. Karoly, 1981: The steady linear response of a spherical atmosphere to thermal and orographic forcing. J. Atmos. Sci., 38, 1179-1196, doi:10.1175/1520-0469(1981)038<1179: TSLROA $>2.0 . \mathrm{CO} ; 2$. 
, and T. Ambrizzi, 1993: Rossby wave propagation on a realistic longitudinally varying flow. J. Atmos. Sci., 50, 1661-1671, doi:10.1175/1520-0469(1993)050<1661:RWPOAR > 2.0.CO;2.

- , and T. Woollings, 2015: Persistent extratropical regimes and climate extremes. Curr. Climate Change Rep., 1 (3), 115-124, doi:10.1007/s40641-015-0020-8.

Kornhuber, K., V. Petoukhov, S. Petri, S. Rahmstorf, and D. Coumou, 2017: Evidence for wave resonance as a key mechanism for generating high-amplitude quasi-stationary waves in boreal summer. Climate Dyn., doi:10.1007/ s00382-016-3399-6, in press.

Lau, W. K. M., and K.-M. Kim, 2012: The 2010 Pakistan flood and Russian heat wave: Teleconnection of hydrometeorological extremes. J. Hydrometeor., 13, 392-403, doi:10.1175/ JHM-D-11-016.1.

Lucarini, V., S. Calmanti, A. Dell'Aquila, P. M. Ruti, and A. Speranza, 2007: Intercomparison of the Northern Hemisphere winter mid-latitude atmospheric variability of the IPCC models. Climate Dyn., 28, 829-848, doi:10.1007/ s00382-006-0213-x.

Lutsko, N. J., and I. M. Held, 2016: The response of an idealized atmosphere to orographic forcing: Zonal vs meridional propagation. J. Atmos. Sci., 73, 3701-3718, doi:10.1175/JAS-D-16-0021.1.

Manola, I., F. Selten, H. de Vries, and W. Hazeleger, 2013: "Waveguidability" of idealized jets. J. Geophys. Res., 118, 10 432-10 440, doi:10.1002/jgrd.50758.

Murdock, J. A., 1987. Perturbations: Theory and Methods. Society for Industrial and Applied Mathematics, $529 \mathrm{pp}$.

Ogi, M., K. Yamazaki, and Y. Tachibana, 2005: The summer northern annular mode and abnormal summer weather in 2003. Geophys. Res. Lett., 32, L04706, doi:10.1029/ 2004GL021528.

O'Kane, T. J., J. S. Risbey, D. P. Monselesan, I. Horenko, and C. L. E. Franzke, 2016: On the dynamics of persistent states and their secular trends in the waveguides of the Southern Hemisphere troposphere. Climate Dyn., 46, 3567-3597, doi:10.1007/s00382-015-2786-8.

Parker, T. J., G. J. Berry, and M. J. Reeder, 2014: The structure and evolution of heat waves in southeastern Australia. J. Climate, 27, 5768-5785, doi:10.1175/JCLI-D-13-00740.1.

Pedlosky, J., 1979: Geophysical Fluid Dynamics. Springer-Verlag, 624 pp.

Peixoto, J. P., and A. H. Oort, 1992: Physics of Climate. American Institute of Physics Press, $520 \mathrm{pp}$.

Pena-Ortiz, C., D. Gallego, P. Ribera, P. Ordonez, and M. D. C. Alvarez-Castro, 2013: Observed trends in the global jet stream characteristics during the second half of the 20th century. J. Geophys. Res. Atmos., 118, 2702-2713, doi:10.1002/jgrd.50305.

Petoukhov, V., S. Rahmstorf, S. Petri, and H. J. Schellnhuber, 2013: Quasiresonant amplification of planetary waves and recent
Northern Hemisphere weather extremes. Proc. Natl. Acad. Sci. USA, 110, 5336-5341, doi:10.1073/pnas.1222000110.

, S. Petri, S. Rahmstorf, D. Coumou, K. Kornhuber, and H. J. Schellnhuber, 2016: Role of quasi-resonant planetary wave dynamics in recent boreal spring-to-autumn extreme events. Proc. Natl. Acad. Sci. USA, 113, 6862-6867, doi:10.1073/ pnas.1606300113.

Pezza, B. A., P. van Rensch, and W. Cai, 2012: Severe heat waves in southern Australia: Synoptic climatology and large scale connections. Climate Dyn., 38, 209-224, doi:10.1007/ s00382-011-1016-2.

Rossby, C.-G., 1939: Relation between variations in the intensity of the zonal circulation of the atmosphere and the displacements of the semi-permanent centers of action. J. Mar. Res., 2, 38-55.

_ 1940: Planetary flow patterns in the atmosphere. Quart. J. Roy. Meteor. Soc., 66, 68-87.

Schneider, T., T. Bischoff, and H. Płotka, 2015: Physics of changes in synoptic midlatitude temperature variability. J. Climate, $\mathbf{2 8}$, 2312-2331, doi:10.1175/JCLI-D-14-00632.1.

Screen, J. A., and I. Simmonds, 2014: Amplified mid-latitude planetary waves favour particular regional weather extremes. Nat. Climate Change, 4, 704-709, doi:10.1038/nclimate2271.

Stadtherr, L., D. Coumou, V. Petoukhov, S. Petri, and S. Rahmstorf, 2016: Record Balkan floods of 2014 linked to planetary wave resonance. Sci. Adv., 2, e1501428, doi:10.1126/ sciadv.1501428.

Teng, H., G. Branstator, H. Wang, G. A. Meehl, and W. M. Washington, 2013: Probability of US heat waves affected by a subseasonal planetary wave pattern. Nat. Geosci., 6, 10561061, doi:10.1038/ngeo1988.

Trenberth, K. E., 1991: Storm tracks in the Southern Hemisphere. J. Atmos. Sci., 48, 2159-2178, doi:10.1175/ 1520-0469(1991)048<2159:STITSH>2.0.CO;2.

Whitham, G. B., 1960: A note on group velocity. J. Fluid Mech., 9, 347-352, doi:10.1017/S0022112060001158.

Woollings, T., 2010: Dynamical influences on European climate: An uncertain future. Philos. Trans. Roy. Soc., 368A, 3733 3756, doi:10.1098/rsta.2010.0040.

Wu, P., 1993: Nonlinear resonance and instability of planetary waves and low-frequency variability in the atmosphere. J. Atmos. Sci., 50, 3590-3607, doi:10.1175/1520-0469(1993)050<3590: NRAIOP $>2.0 . \mathrm{CO} ; 2$.

Yang, S., B. Reinhold, and E. Källén, 1997: Multiple weather regimes and baroclinically forced spherical resonance. J. Atmos. Sci., 54, 1397-1409, doi:10.1175/1520-0469(1997)054<1397: MWRABF $>2.0 . \mathrm{CO} ; 2$.

Zidikheri, M. J., J. S. Frederiksen, and T. J. O'Kane, 2007: Multiple equilibria and atmospheric blocking. Frontiers in Turbulence and Coherent Structures, J. S. Frederiksen and J. Denier, Eds. World Scientific, 59-86. 